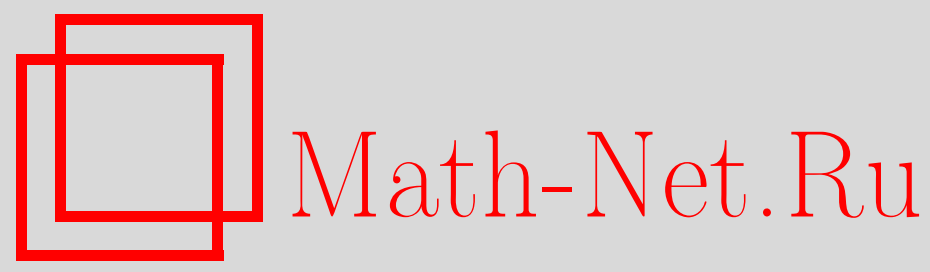

И. С. Сергеев, Быстрые алгоритмы для элементарных операций с комплексными степенными рядами, Дискрет. матем., 2010, том 22, выпуск 1, 17-49

DOI: https://doi.org/10.4213/dm1082

Использование Общероссийского математического портала Math-Net.Ru подразумевает, что вы прочитали и согласны с пользовательским соглашением http://www . mathnet.ru/rus/agreement

Параметры загрузки:

IP : 18.234 .197 .8

26 апреля 2023 г., 10:58:27 


\title{
Быстрые алгоритмы для Элементарных операций с комплексными степенными рядами
}

\author{
() 2010 г. И. С. Сергеев
}

\begin{abstract}
Показано, что инвертирование комплексного степенного ряда может быть выполнено асимптотически со сложностью 5/4 умножений (если сравнивать верхние оценки). Кроме того показано, что извлечение квадратного корня выполняется асимптотически также со сложностью 5/4, вычисление экспоненты - со сложностью $13 / 6$, а возведение в произвольную степень требует 41/12 умножений.

Работа выполнена при поддержке Российского Фонда фундаментальных исследований, проекты 08-01-00863 и 08-01-00632a, программы Президента Российской Федерации поддержки ведущих научных школ, проект НШ 4470.2008.1, и программы фундаментальных исследований Отделения математических наук РАН «Алгебраические и комбинаторные методы математической кибернетики», проект «Синтез и сложность управляющих систем».
\end{abstract}

\section{1. Введение}

В 1966 г. Кук [1] показал, что инвертирование (а следовательно, и деление) чисел может выполняться с такой же по порядку сложностью, как и умножение. Метод Кука является частным случаем дискретного метода последовательных приближений Ньютона-Рафсона, который позволяет вычислять различные элементарные функции, такие как логарифм, экспонента, квадратный корень (см. [2], гл. 9 в [3], [4]).

Метод последовательных приближений работает также и для степенных рядов. В определенном смысле, для степенных рядов он работает даже лучше, чем для чисел: например, логарифм степенного ряда можно вычислить с порядком сложности умножения, а для чисел такого результата получить не удается (см., например, [2]).

Интерес к изучению эффективности метода последовательных приближений применительно к степенным рядам объясняется тем, что степенные ряды представляются теоретически более простым объектом для анализа, кроме того, сегодня арифметика степенных рядов (многочленов) приобретает прикладное значение.

В 1972 г. Зивекинг [5] опубликовал метод Кука для степенных рядов, вероятно, не зная о работе [1]. Впоследствии различные оценки эффективности метода последовательных приближений были получены Брентом и Кунгом в $[2,6]$ и других работах этих авторов.

Практический и теоретический интерес представляет минимизация мультипликативных констант в оценках сложности основных операций со степенными рядами (их удобно соотносить со сложностью базовой операции умножения). Так, Брент [2] указывает, что инвертирование, деление, извлечение квадратного корня, логарифмирование, вычисление 
экспоненты и возведение в степень с произвольным показателем выполняются асимптотически не сложнее, чем $3,4,11 / 2,4,22 / 3$ и 34/3 умножений соответственно (сравнивается сложность операций со степенными рядами по модулю $x^{n}$ ).

Эти оценки можно уточнить, считая, что умножение выполняется специальным способом. В таком случае можно использовать промежуточные результаты вычислений. Над кольцами, содержащими необходимое число корней из единицы (например, над С), умножение рядов естественно выполнять при помощи дискретного преобразования Фурье (ДПФ) (см., например, [7], гл. 7), то есть асимптотически самым быстрым из известных методов. Для операций, перечисленных в предыдущем абзаце, в этой модели известны оценки, асимптотически эквивалентные сложности $3 / 2,5 / 3,11 / 6,5 / 3,7 / 3$ и 4 умножений (первая оценка доказана в [8, 9], вторая и пятая получены в [10], третья - в [9], четвертая и шестая следуют из методов, изложенных в [2]).

Для умножения над любым кольцом можно использовать метод Карацубы [11], который широко применяется на практике. Сложность операций в модели с методом умножения Карацубы изучалась в работе [12], где, в частности, были получены асимптотические оценки 1,1 и $3 / 4$ сложности умножения для операций инвертирования, деления и извлечения квадратного корня соответственно.

В настоящей работе рассматриваются операции над комплексными (и вещественными) степенными рядами в модели с использованием ДПФ. Стандартным образом результаты могут быть расширены на случай произвольного кольца. Для операций инвертирования, извлечения квадратного корня, вычисления экспоненты и возведения в степень с произвольным показателем получены оценки сложности, асимптотически эквивалентные сложности 5/4, 5/4, 13/6 и 41/12 умножений соответственно.

Основой для получения новых оценок является комбинация нескольких известных приемов и одного нового. Это, с одной стороны, приемы разбиения рядов на блоки, предложенные Бернштейном [9] и ван дер Хувеном [10], с другой - выполнение многократных умножений, как в методе Шёнхаге [8], с третьей - предлагаемый в настоящей работе прием вычислений с использованием двойных ДПФ. Основные результаты работы коротко изложены в [13].

Изложение построено следующим образом. В разделах 2-3 сообщаются необходимые сведения о сложности умножения и методе последовательных приближений. В разделе 4 изложены основные приемы вычислений, в том числе концепция двойных ДПФ (п. 4.2) и блочные методы (п. 4.3). Далее, в разделах 5-9 рассматриваются быстрые алгоритмы инвертирования, деления (в том числе деления многочленов с остатком), извлечения квадратного корня, вычисления экспоненты и возведения в степень. В разделе 10 собраны замечания о сложности решения линейных однородных дифференциальных уравнений (п. 10.1) и о мультипликативной сложности рассмотренных операций (п. 10.2).

\section{2. Умножение}

Для умножения в $\mathbf{C}[[x]]$ по модулю $x^{n}$ справедлива оценка

$$
M(n) \leqslant 6 F(n)+O(n),
$$

где

$$
F(n)=\min _{m \geqslant n} F^{\circ}(m)
$$

a $F^{\circ}(n)-$ сложность ДПФ порядка $n$. 
Известные верхние оценки для $F^{\circ}(n)$ : асимптотически $1,5 n \log n$ (логарифмы здесь и дальше по тексту двоичные) операций над $\mathbf{C}$, или $(34 / 9) n \log n$ операций над $\mathbf{R}(\mathrm{cm} .[4,14])$ в том случае, когда $n=2^{k}$. Добавим, что сложность ДПФ с вещественным набором коэффициентов асимптотически не превосходит (17/9) $n \log n$ операций над $\mathbf{R}$ (см. также [4]). Из наблюдения Шёнхаге [15] следует, что эти оценки остаются в силе и для тех чисел $n$, которые имеют вид

$$
n=2^{k} n_{0}, \quad n_{0}=O(\log n) .
$$

Оценка $1,5 n \log n$ операций над $\mathbf{C}$ может быть также доказана для любого $n$ с использованием ДПФ специального вида (см. [16]). Все перечисленные оценки, следовательно, справедливы для $F(n)$ при любом $n$.

Выражение оценок сложности рассматриваемых ниже операций через $F(n)$ корректно в следующем предположении:

$$
F(a n)-a F(n)=O(a n),
$$

где $a \in \mathbf{N}, a=O(\log n)$. В случае невыполнения данного условия следует $F(n)$ в оценках сложности заменить функцией $\bar{F}(n) \geqslant F(n)$, удовлетворяющей ему.

Далее во всех алгоритмах параметр $n$ должен выбираться в виде (1), что не будет специально оговариваться.

\section{3. Метод последовательных приближений}

Пусть требуется найти первые $n$ членов степенного ряда $g \in \mathbf{C}[[x]]$, который является решением уравнения

$$
\varphi[x, g]=h,
$$

где $\varphi[x, y]-$ степенной ряд переменных $x$ и $y, h$ - степенной ряд переменной $x$, а запись $\psi[f]$ означает подстановку в степенной ряд $\psi$ ряда $f$ вместо переменной.

Для произвольного степенного ряда $f$ введем обозначение

$$
f_{. . n}=f \bmod x^{n} .
$$

Пусть $\Delta g$ обозначает формальную производную ряда $g$, а $\Delta_{x} f-$ производную ряда $f$ нескольких переменных по переменной $x$. Справедлив следующий аналог формулы Тейлора.

Лемма 1. Для произвольных рядов $\varphi \in \mathbf{C}[[x, y]], g, u \in \mathbf{C}[[x]]$, где $u_{. .1}=0$, таких, что либо $g_{. .1}=0$, либо существует $k \in \mathbf{N}$, при котором $\Delta_{y}^{k} \varphi=0$, справедливо равенство

$$
\varphi[x, g+u]=\varphi[x, g]+\left(\Delta_{y} \varphi\right)[x, g] u+\left(\Delta_{y}^{2} \varphi\right)[x, g] u^{2} / 2+\ldots
$$

Доказательство этой леммы может быть восстановлено по гл. 2 в [17], где она приводится в несколько отличной формулировке.

Пусть известно $g_{. . m}$, где $g$ - решение (2). Следующая лемма, которая фактически содержится в [17], показывает, как, зная $g_{. . m}$, получить более точное приближение $g_{. . m+n}$.

Лемма 2. Пусть $\varphi \in \mathbf{C}[[x, y]], g, h \in \mathbf{C}[[x]], \varphi[x, g]=h$, ряды $\varphi$ и g удовлетворяют условиям леммы $1, u\left(\Delta_{y} \varphi\right)_{. .1} \neq 0$. Тогда для $m, n \in \mathbf{N}$ при условии, что либо $m \geqslant n$, либо $\Delta_{y}^{2} \varphi=0$, справедлива формула

$$
g_{. . m+n}=g_{. . m}+\left(h-\varphi\left[x, g_{. . m}\right]\right) . . m+n\left(\left(\Delta_{y} \varphi\right)\left[x, g_{. . n}\right]\right)_{. . n}^{-1} \bmod x^{m+n} .
$$


Доказательство. Положим

$$
g=g_{. . m}+x^{m} g^{\prime}
$$

Условия леммы гарантируют выполнение вытекающего из леммы 1 равенства

$$
\begin{aligned}
\left(\varphi\left[x, g_{. . m}\right]\right)_{. . m+n} & =\left(\varphi\left[x, g-x^{m} g^{\prime}\right]\right) . . m+n \\
& =\varphi[x, g]-\left(\Delta_{y} \varphi\right)[x, g] x^{m} g^{\prime} \bmod x^{m+n} \\
& =h-x^{m}\left(\left(\Delta_{y} \varphi\right)\left[x, g_{. . n}\right]\right) . . n g_{. . n}^{\prime} \bmod x^{m+n} .
\end{aligned}
$$

Следовательно,

$$
\begin{aligned}
& \left(h-\varphi\left[x, g_{. . m}\right]\right)_{. . m+n}\left(\left(\Delta_{y} \varphi\right)\left[x, g_{. . n}\right]\right)_{. . n}^{-1} \bmod x^{m+n} \\
& \quad=x^{m}\left(\left(\Delta_{y} \varphi\right)\left[x, g_{. . n}\right]\right) . . n\left(\left(\Delta_{y} \varphi\right)\left[x, g_{. . n}\right]\right)_{. . n}^{-1} g_{. . . n}^{\prime} \bmod x^{m+n} \\
& \quad=x^{m} g_{. . n}^{\prime} \bmod x^{m+n}
\end{aligned}
$$

что и требовалось доказать. Условие $\left(\Delta_{y} \varphi\right)_{. .1} \neq 0$ необходимо для существования обратного к $\left(\Delta_{y} \varphi\right)\left[x, g_{. . n}\right]$ ряда.

Формула (3) фактически является дискретным вариантом итерации метода касательных Ньютона-Рафсона.

Более подробное изложение метода последовательных приближений можно найти в п. 4.7 в [18], главе 2 в [17], главе 9 в [3].

Рассмотрим следующие известные следствия из леммы 2 , которые будут использоваться далее.

1. Инвертирование. Обратный к ряду $f, f . .1 \neq 0$, степенной ряд $r$ является решением задачи $r f=1$ (в обозначениях (2) $\varphi[x, y]=y f[x], h=1$ ). Из леммы 2 вытекает справедливая при любых $m, n$ формула

$$
r_{. . m+n}=r_{. . m}+r_{. . n}\left(1-r_{. . m} f_{. . m+n}\right) \bmod x^{m+n} .
$$

В [19] указано, что вариант этой формулы применялся еще Шульцем для обращения матриц в работе [20] 1933 года.

2. Деление. Сохраняем и далее обозначение $r=1 / f$. Обозначим через $s$ частное от деления ряда $h$ на $f$, оно является решением задачи $s f=h$ (где $\varphi[x, y]=y f[x]$ ), для которой лемма 2 дает справедливую при любых $m, n$ итерацию

$$
s_{. . m+n}=s_{. . m}+r_{. . n}\left(h_{. . m+n}-s_{. . m} f_{. . m+n}\right) \bmod x^{m+n} .
$$

3. Квадратный корень. Пусть требуется найти ряд $f=\sqrt{h}, h_{. .1}=1$. Заметим, что если добавить в базис операцию извлечения квадратного корня в $\mathbf{C}$, то достаточным является ограничение $h_{. .1} \neq 0$.

Ряд $f$ является решением задачи $f^{2}=h$ (в обозначениях (2) $\varphi[x, y]=y^{2}$ ), для которой из леммы 2 вытекает справедливая при $m \geqslant n$ формула

$$
f_{. . m+n}=f_{. . m}+\frac{\left(h_{. . m+n}-f_{. . m}^{2}\right) r_{. . n}}{2} \bmod x^{m+n} .
$$


4. Логарифм. Через $\mathbf{J} g$ обозначим формальный интеграл ряда $g$ со свободным членом $(\mathbf{J} g)_{. .1}=0$. Как обычно, формальный логарифм $\ln g$ ряда $g, g . .1=1$, определяется как

$$
\ln g=(g-1)-(g-1)^{2} / 2+(g-1)^{3} / 3-\ldots
$$

Брент [2] заметил, что сложность логарифмирования степенного ряда по модулю $x^{n}$ не более чем на $O(n)$ превосходит сложность деления, благодаря соотношению

$$
\ln g=\mathbf{J}(\Delta g / g) .
$$

5. Экспонента. Формальная экспонента $e^{g}$ ряда $g$, где $g . .1=0$, определяется как

$$
e^{g}=1+g+g^{2} / 2 !+g^{3} / 3 !+\ldots
$$

Пусть $f=e^{h}$. Решая задачу $\ln f=h$, где $h . .1=0$, методом леммы 2 (в которой $\varphi[x, y]=\ln y)$, получаем справедливую при $m \geqslant n$ формулу

$$
f_{. . m+n}=f_{. . m}+f_{. . n}\left(h-\ln f_{. . m}\right)_{. . m+n} \bmod x^{m+n} .
$$

6. Возведение в степень. Пусть требуется возвести ряд $h, h . .1=1$, в произвольную степень $C \in \mathbf{C}$. Ряд $f=h^{C}$ может быть найден как решение задачи $\ln f=C \ln h$, для которой лемма 2 (где $\varphi[x, y]=\ln y$ и $C \ln h$ вместо $h$ ) дает верную при $m \geqslant n$ формулу

$$
f_{. . m+n}=f_{. . m}+f_{. . n}\left(C \ln h-\ln f_{. . m}\right) . . m+n \bmod x^{m+n} .
$$

\section{4. Эффективное применение ДПФ}

Напомним, что дискретное преобразование Фурье (ДПФ) порядка $n$ для многочлена $g(x)$ определяется как вектор значений $g(x)$ в точках $\omega_{n}^{k}, k=0, \ldots, n-1$, где $\omega_{n}-$ примитивный корень степени $n$ из единицы в С. Полученному вектору значений можно сопоставить многочлен

$$
g^{*}(x)=\sum_{k=0}^{n-1} g\left(\omega_{n}^{k}\right) x^{k}
$$

степени $n-1$. Справедливо соотношение

$$
n\left(g(x) \bmod \left(x^{n}-1\right)\right)=\sum_{k=0}^{n-1} g^{*}\left(\omega_{n}^{n-1-k}\right) x^{k},
$$

которое позволяет восстановить многочлен минимальной степени, принимающий заданный набор значений в точках $\omega_{n}^{k}$. Соответствующее восстанавливающее преобразование называется обратным ДПФ. Сложность прямого ДПФ порядка $n$ (если оно применяется к многочлену степени $O(n))$ и обратного ДПФ различаются на $O(n)$. Более подробно о ДПФ см. гл. 7 в [7] и гл. 8 в [3].

Прежде чем переходить к изложению алгоритмов, перечислим некоторые приемы, позволяющие в ряде случаев сократить сложность вычислений с применением ДПФ. Через $(g)_{n}^{*}$ будем обозначать вектор значений ДПФ порядка $n$ для многочлена $g(x)$. Будем также пользоваться соглашением, что операции с векторами значений выполняются покомпонентно. 


\section{1. Элементарные приемы}

Произведение двух многочленов $a(x)$ и $b(x), \operatorname{deg} a+\operatorname{deg} b<n$, можно вычислить при помощи трех ДПФ порядка $n$ и $O(n)$ прочих операций. Последовательность вычислений здесь следующая. Вычисляются $(a)_{n}^{*}$ и $(b)_{n}^{*}$ посредством двух ДПФ порядка $n$. Затем вычисляется $(a b)_{n}^{*}=(a)_{n}^{*}(b)_{n}^{*}$. Окончательно, при помощи обратного ДПФ восстанавливается произведение $a(x) b(x)$.

Следующий прием позволяет экономно вычислять часть коэффициентов произведения. Заменим в указанной выше схеме умножения $a$ на $b$ ДПФ порядка $n$ на ДПФ порядка $n-k$, где $k<n / 2$. В результате найдем многочлен

$$
c(x)=a(x) b(x) \bmod \left(x^{n-k}-1\right)=(a b)_{. . n-k}+\left\lfloor a b / x^{n-k}\right\rfloor,
$$

где $\left\lfloor f / x^{m}\right\rfloor$ обозначает $(f-f . . m) / x^{m}$. Очевидно, $n-2 k$ коэффициентов многочлена $c$ (при степенях $x^{k}, \ldots, x^{n-k-1}$ ) совпадают с соответствующими коэффициентами многочлена $a b$. Если дополнительно известны младшие $k$ коэффициентов $a b$, то после вычитания $(a b) . . k$ из правой части (10), определяются оставшиеся коэффициенты произведения:

$$
\left\lfloor a b / x^{n-k}\right\rfloor=(c-a b) . . k .
$$

Свойство линейности ДПФ можно использовать для экономного вычисления выражений вида

$$
h(x)=a(x) b(x)+c(x) d(x)
$$

$($ если $\operatorname{deg}(a b) \approx \operatorname{deg}(c d))$. Вместо того, чтобы умножать $a$ на $b$ и $c$ на $d$ по отдельности, и затем произведения складывать, можно операцию сложения произвести с векторами значений ДПФ и сэкономить одно обратное ДПФ, восстановив многочлен $h$ из вектора значений $(h)_{n}^{*}=(a b)_{n}^{*}+(c d)_{n}^{*}$.

\section{2. Двойное ДПФ}

Отметим еще одно наблюдение. Пусть один и тот же многочлен $g(x)$ является сомножителем в двух произведениях степени $n-1$ и степени $n+m-1$. Очевидный способ вычислений состоит в применении ДПФ порядка $n$ для первого умножения и ДПФ порядка $n+m$ для второго. Сложность вычисления $(g)_{n}^{*}$ и $(g)_{n+m}^{*}$ в данном способе оценивается асимптотически как $F^{\circ}(n)+F^{\circ}(n+m)$.

В некоторых случаях последнюю оценку можно уточнить, выделив общую часть двух ДПФ. Известно [21], что ДПФ составного порядка $k d$ сводится к вычислению $k$ ДПФ порядка $d, d$ ДПФ порядка $k$ и $k d$ умножениям в $\mathbf{C}$, то есть

$$
F^{\circ}(k d) \leqslant k F^{\circ}(d)+d F^{\circ}(k)+O(k d) .
$$

Положим

$$
d=\text { НОД }(n, m) .
$$

Тогда при вычислении $(g)_{n}^{*}$ и $(g)_{n+m}^{*}$ указанным методом одно из ДПФ порядка $d$, а именно то, которое вычисляет $(g)_{d}^{*}$, является общим. В случае $d \asymp m \asymp n$ оценка сложности принимает вид $(2 n+m-d) F^{\circ}(d) / d+O(n+m)$, где символом $\asymp$ обозначается равенство по порядку. Так, в типичном случае $m=n / 2$ тривиальная оценка $5 F^{\circ}(m)$ 
сокращается до $4 F^{\circ}(m)$. Ниже мы покажем, что подходящим образом модифицированная задача может быть решена с асимптотической сложностью $3 F^{\circ}(m)$, а в общем случае фактически со сложностью ДПФ порядка $n+m$.

Рассмотрим следующую модификацию ДПФ, которую мы назовем двойным ДПФ. Вектор значений двойного ДПФ порядка $(n, m)$ определяется следующим образом: первые $n$ компонент образуют вектор значений ДПФ порядка $n$, а остальные $m$ - вектор значений композиции замены переменной $x \rightarrow \zeta x$ и ДПФ порядка $m$, где $\zeta-$ подходящее комплексное число. Другими словами, двойное ДПФ вычисляет вектор значений многочлена $g(x)$ в точках $\omega_{n}^{k}, k=0,1, \ldots, n-1$ и $\zeta \omega_{m}^{k}, k=0,1, \ldots, m-1$, такой вектор будем обозначать через $(g)_{n, m}^{*}$. Для существования всюду определенного обратного преобразования необходимо и достаточно, чтобы все точки в указанном наборе были различны это, как легко проверить, равносильно условию $\zeta \neq 0, \zeta^{\text {НОК }(m, n)} \neq 1$.

Из определения видно, что двойное ДПФ порядка $(n, m)$ может быть выполнено со сложностью $F^{\circ}(n)+F^{\circ}(m)+O(n+m)$. Покажем, что то же справедливо и в отношении обратного преобразования.

Теорема 1. Обратное к двойному ДПФ порядка $(n, m)$ преобразование может быть реализовано за $F^{\circ}(n)+F^{\circ}(m)+O(n+m)$ операций.

Доказательство. Пусть задан вектор значений $(f)_{n, m}^{*}$ двойного ДПФ для многочлена $f$ степени не выше $n+m-1$. Искомые коэффициенты многочлена при соответствующих степенях $x^{i}$ обозначим через $f_{i}$.

Применяя обратное ДПФ порядка $n$ к первым $n$ компонентам вектора $(f)_{n, m}^{*}$, находим многочлен

$$
a(x)=f(x) \bmod \left(x^{n}-1\right) .
$$

Аналогично, применяя обратное ДПФ порядка $m$ к последним $m$ компонентам вектора $(f)_{n, m}^{*}$, восстанавливаем многочлен $f(\zeta x) \bmod \left(x^{m}-1\right)$.

Рассмотрим случай $n \geqslant m$. Подстановка $x / \zeta$ вместо $x$ переводит многочлен $f(\zeta x) \bmod$ $\left(x^{m}-1\right)$ в многочлен

$$
b(x)=f(x) \bmod \left(x^{m}-\zeta^{m}\right) .
$$

Указанная подстановка реализуется со сложностью $O(m)$.

Остается показать, что по остаткам $a(x)$ и $b(x)$ многочлен $f(x)$ может быть вычислен со сложностью $O(n+m)$.

Старшие $n-m$ коэффициентов многочлена $a(x)$ совпадают со средними коэффициентами $f(x)$ (см. выше п. 4.1). Для остальных $2 m$ коэффициентов $f(x)$ (после исключения известных) справедлива система линейных уравнений вида

$$
\left[\begin{array}{ll}
\mathbf{E}_{m} & \mathbf{E}_{m} \\
\mathbf{E}_{m} & \mathbf{T}_{m}
\end{array}\right] F=B,
$$

где $\mathbf{E}_{m}-$ единичная $m \times m$ матрица,

$$
\mathbf{T}_{m}=\left[\begin{array}{cccc} 
& \zeta^{k m} & & \\
& & \ddots & \\
\zeta^{(k+1) m} & & & \zeta^{k m} \\
& \ddots & &
\end{array}\right], \quad F=\left[\begin{array}{c}
f_{0} \\
\vdots \\
f_{m-1} \\
f_{n} \\
\vdots \\
f_{n+m-1}
\end{array}\right]
$$




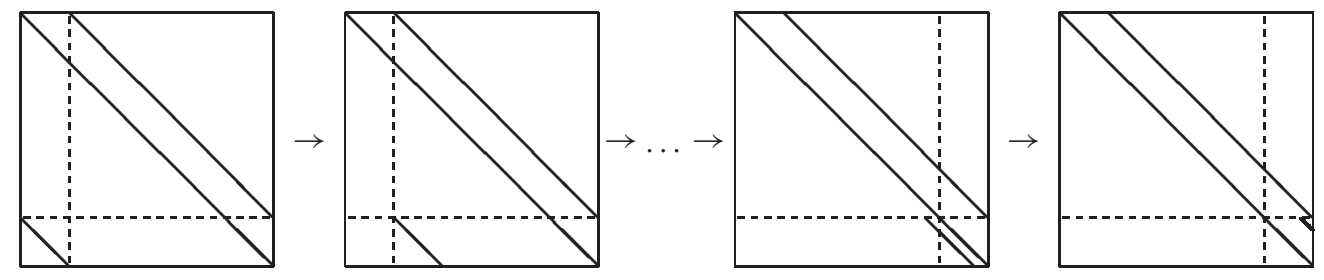

Pис. 1.

(в матрице $\mathbf{T}_{m}$ ненулевой является только одна циклическая диагональ), $f_{i}-$ коэффициент $f(x)$ при $x^{i}, k=\lfloor n / m\rfloor$.

В случае $m \mid n$ матрица $\mathbf{T}_{m}$ является диагональной, и методом Гаусса система (11), очевидно, решается за $O(m)$ операций. В противном случае, исключая из уравнений нижней половины системы (11) первые $m$ неизвестных (для этого требуется выполнить $m$ вычитаний в $\mathbf{C})$, приходим к системе

$$
\left[\begin{array}{cc}
\mathbf{E}_{m} & \mathbf{E}_{m} \\
0 & \mathbf{Z}_{m}
\end{array}\right] F=B^{\prime},
$$

где

$$
\mathbf{Z}_{m}=\mathbf{T}_{m}-\mathbf{E}_{m}
$$

Для краткости будем говорить, что матрица имеет тип $Z$, если у нее все элементы, расположенные за пределами главной и одной из параллельных ей циклических диагоналей, равны нулю. В частности, матрица $\mathbf{Z}_{m}$ имеет тип $Z$.

После разрешения подсистемы системы (12) с матрицей $\mathbf{Z}_{m}$ относительно переменных $f_{n}, \ldots, f_{n+m-1}$, оставшиеся неизвестные могут быть найдены за $O(m)$ операций.

Лемма 3. Сложность решения системы линейных уравнений с $n \times n$ матрицей типа $Z$ составляет $O(n)$ (подразумевается существование решения).

Доказательство. Пусть $k$ - число элементов циклической диагонали, расположенных под главной диагональю матрицы. Без ограничения общности предположим, что $k \leqslant n / 2$. В случае $k>n / 2$ достаточно перейти к эквивалентной системе с транспонированной матрицей и перевернутыми векторами неизвестных и свободных членов.

Методом Гаусса будем последовательно исключать крайние левые вхождения ненулевых элементов в нижних $k$ строках. Один проход этих строк выполняется со сложностью $O(k)$. При этом диагональ с ненулевыми элементами перемещается на $k$ позиций вправо. Выполняется $\lfloor n / k\rfloor$ таких шагов: на последнем шаге исключение применяется только к элементам, расположенным за пределами правого нижнего квадрата размера $k \times k$. Описанный процесс схематически изображен на рис. 1 (диагональными линиями отмечены вхождения ненулевых элементов). По построению, в результате правая нижняя подматрица размера $k \times k$ будет иметь тип $Z$.

При условии разрешения полученной подсистемы относительно последних $k$ переменных оставшиеся неизвестные вычисляются со сложностью $O(n)$. Обозначая через $T(n)$ функцию сложности решения системы с матрицей размера $n \times n$, получаем рекуррентное неравенство

$$
T(n) \leqslant T(k)+O(n) \leqslant T(n / 2)+O(n),
$$


из которого следует, что

$$
T(n)=O(n)
$$

Лемма доказана.

Доказательством леммы завершается рассмотрение случая $n \geqslant m$ теоремы 1 . В случае $n<m$ строим систему линейных уравнений относительно коэффициентов многочлена $f(\zeta x)$, предварительно вычислив

$$
\left.\left(f(x) \bmod \left(x^{n}-1\right)\right)\right|_{x \rightarrow \zeta x}=f(\zeta x) \bmod \left(x^{n}-\zeta^{-n}\right) .
$$

К полученной системе применимы проведенные для первого случая рассуждения. Теорема 1 доказана.

Таким образом, вместо вычисления $(g)_{n}^{*}$ и $(g)_{n+m}^{*}$ в примере из начала пункта можно вычислить $(g)_{n, m}^{*}$ со сложностью $F^{\circ}(n)+F^{\circ}(m)+O(n+m)$ (то есть асимптотически $3 F^{\circ}(m)$ в случае $\left.m=n / 2\right)$.

Двойное ДПФ не обладает указанным в п. 4.1 свойством обычного ДПФ неполного порядка вычислять часть средних коэффициентов произведения. Однако справедлив более слабый аналог этого свойства.

Пусть

$$
\operatorname{deg} a+\operatorname{deg} b<n
$$

и известны младшие $k$ коэффициентов произведения $a(x) b(x)$. Тогда остальные коэффициенты произведения можно вычислить при помощи трех двойных ДПФ (двух прямых и одного обратного) порядка $\left(n^{\prime}, m^{\prime}\right)$ и $O(n)$ прочих операций, где

$$
n^{\prime}+m^{\prime}=n-k, \quad k \leqslant \min \left\{m^{\prime}, n^{\prime}\right\} .
$$

Действительно, положим

$$
a(x) b(x)=(a b)_{. . n-k}+x^{n-k} h(x), \quad \operatorname{deg} h<k .
$$

Тогда

$$
\begin{aligned}
c(x) & =a(x) b(x) \bmod \left(x^{n^{\prime}}-1\right)\left(x^{m^{\prime}}-\zeta^{m^{\prime}}\right) \\
& =(a b) . . n-k-h(x)\left(\zeta^{m^{\prime}} x^{n^{\prime}}+x^{m^{\prime}}-\zeta^{m^{\prime}}\right) .
\end{aligned}
$$

Зная $c(x)$ и $(a b) . . k$, можно найти

$$
h(x)=\zeta^{-m^{\prime}}(c-a b) . . k,
$$

а затем и остальные коэффициенты многочлена $a b$.

Для нас далее будет важно уточнение этого свойства в случае, когда двойное ДПФ порядка $(2 n, n)$ используется для нахождения коэффициентов многочлена степени не выше $5 n-1$. 
Лемма 4. Пусть известен вектор значений двойного ДПФ порядка $(2 n, n)$ для многочлена $f, \operatorname{deg} f<5 n$, и также известен многочлен $f_{\text {..2n. }}$. Тогда коэффищиенты $f$ могут быть вычислены за $F^{\circ}(2 n)+F^{\circ}(n)+O(n)$ операчий.

Доказательство. Применяя обратное ДПФ порядка $(2 n, n)$, находим многочлен

$$
c(x)=f(x) \bmod \left(x^{2 n}-1\right)\left(x^{n}-\xi^{n}\right) .
$$

Очевидно, коэффициент многочлена $c(x)$ при $x^{j n+i}$, где $j=0,1,2$, а $i=0,1, \ldots, n-1$, является линейной комбинацией коэффициентов $f_{j n+i}, f_{3 n+i}$ и $f_{4 n+i}$ (через $f_{k}$ обозначается коэффициент $f(x)$ при $x^{k}$ ). Таким образом, получаем систему линейных уравнений относительно $3 n$ неизвестных коэффициентов многочлена $f(x)$, которая имеет вид

$$
\left[\begin{array}{ccc}
\mathbf{E}_{n} & \mathbf{D}_{n}^{1} & \mathbf{D}_{n}^{2} \\
0 & \mathbf{D}_{n}^{3} & \mathbf{D}_{n}^{4} \\
0 & \mathbf{D}_{n}^{5} & \mathbf{D}_{n}^{6}
\end{array}\right] F=B, \quad F=\left[\begin{array}{c}
f_{2 n} \\
\vdots \\
f_{5 n-1}
\end{array}\right],
$$

где $\mathbf{D}_{n}^{i}, i=1, \ldots, 6,-$ диагональные $n \times n$ матрицы. Для решения указанной системы методом Гаусса достаточно $O(n)$ операций. Лемма доказана.

По аналогии с двойным ДПФ можно определить и тройное ДПФ, и ДПФ большей кратности. Например, вектор значений тройного ДПФ порядка $(n, m, p)$ определяется следующим образом: первые $n+m$ компонент образуют вектор значений двойного ДПФ порядка $(n, m)$, а остальные $p$ - вектор значений композиции замены переменной $x \rightarrow \eta x$ и ДПФ порядка $p$, где $\eta$ - подходящее комплексное число.

В действительности, далее будет использоваться только тройное ДПФ порядка $(2 n, n, n)$. Несложно проверить, что обратное к нему преобразование можно выполнить за $F^{\circ}(2 n)+2 F^{\circ}(n)+O(n)$ операций. На самом деле, при выборе параметров $\zeta=\omega_{4 n}$ и $\eta=\omega_{4 n}^{3}$ указанное тройное ДПФ с точностью до перестановки компонент вектора значений совпадает с ДПФ порядка $4 n$, что позволяет избежать накладных расходов, связанных с решением системы линейных уравнений.

Аналогично, вместо наиболее употребимого далее двойного ДПФ порядка $(2 n, n)$, возможно, иногда целесообразно использовать тройное ДПФ порядка $(n, n, n)$ с параметрами $\zeta=\omega_{3 n}$ и $\eta=\omega_{3 n}^{2}$, поскольку такое ДПФ с точностью до перестановки компонент вектора значений совпадает с ДПФ порядка $3 n$.

\section{3. Разбиение на блоки}

Два эффективных способа организации итерационных вычислений со степенными рядами, в которых используется подходящее разбиение рядов на блоки, были разработаны Бернштейном [9] и ван дер Хувеном [10]. Далее для краткости эти способы мы будем называть методом $A$ и методом $Б$ соответственно.

\subsection{1. Метод $A$}

Рассмотрим следующий пример. Пусть требуется перемножить два многочлена (степенных ряда) $f$ и $g$ по модулю $x^{k n}$. Разобьем $f$ и $g$ на блоки длины $n$ :

$$
\begin{aligned}
& f=a_{0}+a_{1} x^{n}+a_{2} x^{2 n}+\ldots, \\
& g=b_{0}+b_{1} x^{n}+b_{2} x^{2 n}+\ldots,
\end{aligned}
$$


где $\operatorname{deg} a_{i}, b_{i}<n$. Тогда

$$
f g=c_{0}+c_{1} x^{n}+c_{2} x^{2 n}+\ldots
$$

где

$$
c_{i}=\sum_{\lambda+\mu=i} a_{\lambda} b_{\mu}
$$

и $\operatorname{deg} c_{i}<2 n$. Вычисление можно произвести следующим образом. При помощи $2 k$ ДПФ порядка $2 n$ найти все $\left(a_{i}\right)_{2 n}^{*}$ и $\left(b_{i}\right)_{2 n}^{*}, i<k$. Опираясь на свойство линейности ДПФ, по формуле (15) вычислить все $\left(c_{i}\right)_{2 n}^{*}$, что выполняется за $O(n M(k))$ операций (так как вектор значений многочленов $c_{i}$ в фиксированной точке является сверткой векторов значений $a_{i}$ и $b_{i}$ в той же точке). Далее находятся все многочлены $c_{i}, i<k$, посредством $k$ обратных ДПФ порядка $2 n$. Окончательно, многочлен $f g \bmod x^{k n}$ восстанавливается по формуле (14) за $O(k n)$ операций. Общая сложность вычислений оценивается как $3 k F^{\circ}(2 n)+O(n k \log k)$. (В [9] указан худший остаточный член ввиду реализации свертки стандартным алгоритмом.)

Такой способ вычислений будем называть методом $A$, более конкретно, методом $A-2 n$, в соответствии с порядком используемых ДПФ. В примере выше он асимптотически не дает выигрыша по сравнению со стандартным методом сложности $3 F^{\circ}(2 k n)+O(k n)$, однако применение указанного приема внутри итерационного процесса может приводить к экономии за счет многократного использования результатов промежуточных вычислений. По аналогии можно рассматривать также метод $A-3 n$, предназначенный для вычисления тройных произведений, и метод $A-(2 n, n)$, в котором используются двойные ДПФ порядка $(2 n, n)$, эффективные для тех алгоритмов, где выполняются как обычные, так и тройные умножения.

\subsection{2. Метод $Б$}

Вернемся к примеру умножения степенных рядов $f$ и $g$ по модулю $x^{k n}$. Пусть

$$
f g=d_{0}+d_{1} x^{n}+d_{2} x^{2 n}+\ldots,
$$

где $\operatorname{deg} d_{i}<n$. Предположим, что нам надо вычислить не все $d_{i}, i<k$, а только один блок $d_{j}$. Поступая как выше (см. (14)), мы восстанавливаем $c_{j-1}$ и $c_{j}$ при помощи двух ДПФ порядка $2 n$ и затем находим

$$
d_{j}=\left\lfloor c_{j-1} / x^{n}\right\rfloor+c_{j} \bmod x^{n} .
$$

Ван дер Хувен [10] заметил, что одно ДПФ можно сэкономить, если воспользоваться формулой

$$
d_{j}=\left\lfloor\left(\xi_{j} b_{0}+\xi_{j-1} b_{1}+\ldots+\xi_{0} b_{j}\right) / x^{n}\right\rfloor \bmod x^{n}
$$

где $\xi_{0}=a_{0} x^{n}$ и $\xi_{i}=a_{i-1}+a_{i} x^{n}$ при $i>0$, а $a_{i}$ и $b_{i}$ взяты из (13). Вычисление по формуле (16) состоит в нахождении средних $n$ коэффициентов суммы $\sum \xi_{\lambda} b_{j-\lambda}$ (при степенях $\left.x^{n}, \ldots, x^{2 n-1}\right)$, которая является многочленом степени $3 n-2$, поэтому применение ДПФ порядка $2 n$ корректно, см. (10).

В рамках той же задачи умножения рядов $f$ и $g$ по модулю $x^{k n}$ дополнительно предположим, что блоки $a_{j}$ и $b_{j}$ становятся известными только после того, как вычислен 
очередной блок $d_{j-1}$ произведения $f g$. Последовательное вычисление $d_{j}, j=0, \ldots, k-1$, требует $3 k F^{\circ}(2 n)+O\left(k^{2} n\right)$ операций. На $j$-м шаге выполняется по три ДПФ порядка $2 n$ и $O(j n)$ операций при вычислении $\left(\sum \xi_{\lambda} b_{j-\lambda}\right)_{2 n}^{*}$ по формуле (16). Метод работы [22] позволяет указать остаточный член в виде $O\left(k n e^{2 \sqrt{\log \log k}} \log k\right)$. Если $k-$ слабо растущая относительно $n$ функция, то произведение вычисляется асимптотически со сложностью стандартного способа.

Ван дер Хувен [10] предложил этот метод (назовем его методом 5 или $5-2 n)$ для алгоритмов, основанных на методе последовательных приближений, и продемонстрировал его эффективность на примерах задач деления и решения линейных однородных дифференциальных уравнений, в частности, вычисления экспоненты.

Метод $Б$ несложно распространить на вычисление тройных произведений, используя ДПФ порядка $3 n$. Пусть вычисляется произведение степенных рядов $f, g$ и $h$, которые разбиты на блоки $a_{i}, b_{i}$ и $c_{i}$ длины $n$ соответственно. Положим

$$
f g h=d_{0}+d_{1} x^{n}+d_{2} x^{2 n}+\ldots,
$$

где $\operatorname{deg} d_{i}<n$.

Для $i \geqslant 0$ введем обозначение

$$
\xi_{i}=a_{i-2}+a_{i-1} x^{n}+a_{i} x^{2 n},
$$

полагая $a_{-2}=a_{-1}=0$. Пусть

$$
\begin{aligned}
\gamma_{j} & =\sum_{\lambda+\mu+\nu=j} a_{\lambda} b_{\mu} c_{\nu}, \\
\delta_{j} & =\sum_{\lambda+\mu+\nu=j} \xi_{\lambda} b_{\mu} c_{\nu} .
\end{aligned}
$$

В силу определения многочленов $\xi_{i}$, справедливо равенство

$$
\delta_{j}=\gamma_{j-2}+\gamma_{j-1} x^{n}+\gamma_{j} x^{2 n},
$$

где полагается $\gamma_{-2}=\gamma_{-1}=0$. Учитывая, что

$$
d_{j}=\gamma_{j}+\left\lfloor\gamma_{j-1} / x^{n}\right\rfloor+\left\lfloor\gamma_{j-2} / x^{2 n}\right\rfloor \bmod x^{n},
$$

получаем равенство

$$
d_{j}=\left\lfloor\delta_{j} / x^{2 n}\right\rfloor \bmod x^{n},
$$

то есть коэффициенты многочлена $d_{j}$ совпадают с коэффициентами многочлена $\delta_{j}$ при степенях $x^{2 n}, \ldots, x^{3 n-1}$. Следовательно (см. п. 4.1), они могут быть восстановлены из вектора значений ДПФ порядка $3 n$ для многочлена $\delta_{j}$.

Указанное в п. 4.2 ограничение, присущее двойному ДПФ, не позволяет использовать вместо ДПФ порядка $3 n$ двойное ДПФ порядка $(2 n, n)$ в вышеприведенной схеме вычислений. Это ограничение, впрочем, можно преодолеть в случае, когда задача состоит в последовательном нахождении всех $d_{j}$, начиная с $j=0$.

Действительно, согласно (17), младшие $2 n$ коэффициентов многочлена $\delta_{j}$ зависят только от коэффициентов многочленов $\gamma_{j-1}$ и $\gamma_{j-2}$. Исходя из (17), несложно проверить, что многочлен $\gamma_{j}$ выражается через многочлены $\delta_{i}, i \leqslant j$. Следовательно, младшие $2 n$ коэффициентов многочлена $\delta_{j}$ могут быть найдены, если известны многочлены $\delta_{i}$ (или $\gamma_{i}$ ) при $i<j$. Поэтому, согласно лемме 4 , многочлен $\delta_{j}$ может быть восстановлен целиком 
из вектора значений двойного ДПФ порядка $(2 n, n)$ при условии, что многочлены $\delta_{i}$ (или $\left.\gamma_{i}\right)$, где $i<j$, вычислены.

Таким образом, в случае использования двойных ДПФ порядка $(2 n, n)$ рассматриваемую схему вычислений достаточно дополнить вычислением многочленов $\delta_{j}$ (или $\left.\gamma_{j}\right)$.

Остаточный член в оценке сложности вычисления блоков $d_{j}, j=0,1, \ldots, k-1$, можно указать в виде $O\left(k^{2} n\right)$. Он определяется вычислением векторов значений ДПФ для многочленов $\delta_{j}$ на $j$-м шаге, для этого требуется выполнить $O(j n)$ операций.

\subsection{3. Тройное умножение специального вида}

Рассмотрим задачу вычисления блоков $d_{i}$ в произведении вида

$$
f\left\lfloor g h / x^{l n}\right\rfloor=d_{0}+d_{1} x^{n}+d_{2} x^{2 n}+\ldots,
$$

где степенные ряды $f, g$ и $h$ разбиты на блоки $a_{i}, b_{i}$ и $c_{i}$ длины $n$ соответственно. Укажем способ, не использующий вычисления произведения $g h$ и подходящий для реализации методом $A-3 n$ или $A-(2 n, n)$. В некоторых случаях такой способ позволяет получить выигрыш, что будет видно на примере алгоритма вычисления экспоненты из п. 8.2.

Осложняющим обстоятельством для применения блочных методов в данном случае является своего рода граничный эффект, возникающий от укорачивания внутреннего произведения $g h$. С целью учета этого эффекта положим

$$
\theta=\left\lfloor\sum_{\lambda+\mu=l-1} b_{\lambda} c_{\mu} / x^{n}\right\rfloor
$$

Тогда

$$
\left\lfloor g h / x^{l n}\right\rfloor=u_{0}+u_{1} x^{n}+u_{2} x^{2 n}+\ldots
$$

где

$$
u_{0}=\theta+\sum_{\lambda+\mu=l} b_{\lambda} c_{\mu}, \quad u_{i}=\sum_{\lambda+\mu=l+i} b_{\lambda} c_{\mu}, \quad i>0 .
$$

Теперь искомое произведение может быть найдено как

$$
f\left\lfloor g h / x^{l n}\right\rfloor=\delta_{0}+\delta_{1} x^{n}+\delta_{2} x^{2 n}+\ldots, \quad \delta_{i}=\sum_{\lambda+\mu=i} a_{\lambda} u_{\mu},
$$

где $\operatorname{deg} \delta_{i}<3 n$. Таким образом, для вычисления многочленов $\delta_{i}$ можно использовать ДПФ порядка $3 n$ или двойные ДПФ порядка $(2 n, n)$.

В тех случаях, когда требуется вычислить один первый блок длины $n$ произведения (18) (как в методе Б), мы будем применять разбиение рядов на блоки длины $o(n)$ и проводить вычисления в духе метода $A$. Пример алгоритма, в котором используется этот прием, будет предъявлен в разделе 9 для реализации возведения в степень.

Указанная техника позволяет вычислять блок произведения вида (18) фактически с такой же сложностью, как и блок обычного тройного произведения $f g h$.

Ниже будет показано, что методы, основанные на блочном разбиении, являются эффективными с асимптотической точки зрения. Бернштейн [9] однако отмечает, что они (имелся в виду метод $A$, но, вероятно, то же справедливо и в отношении метода $Б$ ) не столь эффективны с точки зрения прикладных вычислений (остаточный член вносит весомый вклад в сложность при малых значениях $n$ ). Поэтому в большинстве случаев мы также будем уделять внимание модификациям стандартного метода, основанным на материале п. 4.1, 4.2. 


\section{5. Инвертирование}

Для нахождения обратного к ряду $f$ степенного ряда $r$ обычно (например, $[1,8,9])$ используется итерация второго порядка (то есть итерация, удваивающая точность)

$$
r_{. .2 n}=r_{. . n}\left(2-r_{. . n} f_{.2 n}\right) \bmod x^{2 n}
$$

которая является частным случаем (4) при $m=n$.

Быстрые варианты метода Кука [1], предложенные независимо Шёнхаге [8] и Бернштейном [9] в 2000 г., находят $r_{. . n}$, используя приблизительно $9 F(n)$ операций. Метод $A-3 n$ позволяет сократить объем вычислений до 7,5F $(n)$. Обозначим через $I(n)$ сложность инвертирования по модулю $x^{n}$.

Теорема 2. Справедливы соотношения

(a) $I(n) \leqslant 9 F(n)+O(n)$,

(б) $I(n) \leqslant 7,5 F(n)+O(n \log \log n)$.

\section{1. Алгоритм Шёнхаге}

В методе Шёнхаге [8] итерация (19) реализуется следующим образом:

(0) Пусть многочлен $r_{. . n}$ вычислен.

(1) Вычислить $\left(r_{. . n}\right)_{3 n}^{*}$ и $\left(f_{. .2 n}\right)_{3 n}^{*}$.

Введем обозначение

$$
\alpha=r_{. . n}\left(1-r_{. . n} f_{. .2 n}\right)=x^{n} \beta .
$$

(2) Вычислить $(\alpha)_{3 n}^{*}=\left(r_{. . n}\right)_{3 n}^{*}\left(1-\left(r_{. . n}\right)_{3 n}^{*}\left(f_{.2 n}\right)_{3 n}^{*}\right)$.

(3) Вычислить $\alpha \bmod \left(x^{3 n}-1\right)=\left\lfloor\beta / x^{2 n}\right\rfloor+x^{n} \beta . .2 n$, откуда находится $\beta$. Тогда

$$
r_{.2 n}=r_{. . n}+x^{n} \beta_{. . n} .
$$

Для выполнения шагов 1, 3 требуется три ДПФ порядка $3 n$, остальные действия выполняются за $O(n)$ операций. Из рекуррентного соотношения

$$
I(2 n) \leqslant 3 F^{\circ}(3 n)+I(n)+O(n)
$$

вытекает оценка

$$
I(n) \leqslant 9 F(n)+O(n) .
$$

Метод $A-2 n$, который использовал Бернштейн [9], приводит к оценке

$$
I(n) \leqslant 9 F(n)+O(n \log \log n),
$$

где остаточный член по порядку хуже, чем в методе Шёнхаге. 


\section{2. Асимптотически эффективный метод}

Вторую часть теоремы 2 доказывает алгоритм, основанный на методе $A-3 n$. На примере алгоритма Шёнхаге мы видим, что ДПФ порядка $3 n$ применяется к $r . . n$, а на следующем шаге ДПФ порядка $6 n$ применяется к $r . .2 n$ (то же самое с $f_{. .2 n}$ ). Эти вычисления имеют общую часть, которая в блочном методе реализуется отдельно.

Представим $f$ и $r$ в виде

$$
\begin{aligned}
& f=a_{0}+a_{1} x^{n}+a_{2} x^{2 n}+\ldots, \\
& r=b_{0}+b_{1} x^{n}+b_{2} x^{2 n}+\ldots,
\end{aligned}
$$

где $\operatorname{deg} a_{i}, b_{i}<n$. Введем обозначение

$$
\xi_{i}=a_{i-2}+a_{i-1} x^{n}+a_{i} x^{2 n},
$$

где $a_{-2}=a_{-1}=0$.

Выберем $k \in \mathbf{N}$ и рассмотрим следующий алгоритм.

(0) Пусть многочлен $r_{. . n}=b_{0}$ известен.

$(0.1)$ Вычисляются $\left(\xi_{0}\right)_{3 n}^{*}$ и $\left(b_{0}\right)_{3 n}^{*}$.

Далее на каждом $i$-м шаге, $i=1, \ldots, k$, посредством (19) находится $r_{. .2^{i} n}$. Используется метод тройного умножения, описанный в п. 4.3.2.

(i.1) В случае $i>1$ вычисляются $\left(b_{j}\right)_{3 n}^{*}$ для всех $j=2^{i-2}, \ldots, 2^{i-1}-1$.

(i.2) Вычисляются $\left(\xi_{j}\right)_{3 n}^{*}$ для всех $j=2^{i-1}, \ldots, 2^{i}-1$. Пусть

$$
\alpha=r_{. .2^{i-1} n}\left(1-r_{. .2^{i-1} n} f_{. .2^{i} n}\right)=x^{2^{i-1} n} \beta .
$$

Представим $\beta$ в виде

$$
\beta=c_{0}+c_{1} x^{n}+c_{2} x^{2 n}+\ldots,
$$

где $\operatorname{deg} c_{j}<n$. Введем обозначение

$$
\gamma_{j}=-\sum_{\substack{\lambda+\mu+\nu=j+2^{i-1} \\ \lambda, \mu<2^{i-1}}} b_{\lambda} b_{\mu} \xi_{\nu}
$$

Очевидно,

$$
\operatorname{deg} \gamma_{j}<5 n
$$

(i.3) Вычисляются $\left(\gamma_{j}\right)_{3 n}^{*}, j=0,1, \ldots, 2^{i-1}-1$, исходя из (20).

(i.4) Вычисляются $\gamma_{j} \bmod \left(x^{3 n}-1\right), j=0,1, \ldots, 2^{i-1}-1$. Находятся блоки $c_{j}$, образованные средними $n$ коэффициентами $\gamma_{j}$, то есть

$$
c_{j}=\left\lfloor\gamma_{j} / x^{2 n}\right\rfloor \bmod x^{n},
$$

после чего становится известным $\beta_{. .2^{i-1} n}$. Окончательно получаем, что

$$
r_{. .2^{i} n}=r_{. .2^{i-1} n}+x^{2^{i-1} n} \beta_{. .2^{i-1} n} .
$$


Оценим сложность вычислений на $i$-м шаге. В п. $(i .1),(i .2)$ и $(i .4)$ выполняется всего $2^{i}+2^{i-2}$ ДПФ порядка $3 n$. П. (i.3) выполняется со сложностью $O\left(n M\left(2^{i}\right)\right)($ см. п. 4.3.1), а остальные действия - со сложностью $O\left(2^{i} n\right)$. Суммируя оценки сложности по всем шагам, получаем оценку

$$
I\left(2^{k} n\right) \leqslant\left(2,5 \cdot 2^{k}-1\right) F^{\circ}(3 n)+I(n)+O\left(2^{k} k n\right) .
$$

Выбирая

$$
k=\log \log n+O(1)
$$

получаем, что

$$
I(n) \leqslant 7,5 F(n)+O(n \log \log n) .
$$

Заметим, и будем пользоваться этим наблюдением далее, что в приведенных алгоритмах инвертирования ДПФ порядка $3 n$ можно заменить на двойные ДПФ порядка $(2 n, n)$.

\section{6. Деление}

Рассмотрим задачу нахождения частного $s$ от деления степенного ряда $h$ на ряд $f$ по модулю $x^{n}$, сохраняя обозначение $r=1 / f$. Сложность этой операции обозначим через $D(n)$. Один из экономных способов деления был предложен Карпом и Маркштейном [23]. Он основан на стандартном алгоритме инвертирования, в последнюю итерацию которого встроено умножение на $h$. Быстрый вариант алгоритма [23], использующий асимптотически $12,5 F(n)$ операций, описан в [12]. Эта оценка может быть уменьшена до $12 F(n)$ при помощи идеи из [24]. Ван дер Хувен [10] при помощи метода $5-2 n$ улучшил оценку сложности деления до $10 F(n)$ (см. [10]).

Теорема 3. Справедливы соотношения

(a) $D(n) \leqslant 12 F(n)+O(n)$,

(б) $D(n) \leqslant 10 F(n)+O(n \sqrt{\log n})$.

\section{1. Алгоритм Карпа-Маркштейна}

Первую часть теоремы доказывает метод [23, 12], основанный на формуле

$$
s_{. .2 n}=s_{. . n}+r_{. . n}\left(h_{.2 n}-s_{. . n} f_{.2 n}\right) \bmod x^{2 n},
$$

которая вытекает из (5) при $m=n$.

Опишем алгоритм $[23,12]$.

(0) Пусть многочлен $r_{. . n}$ вычислен.

(1) Вычислить $\left(r_{. . n}\right)_{2 n}^{*},\left(f_{. .2 n}\right)_{2 n}^{*},(h . . n)_{2 n}^{*}$.

Пусть $\beta=r_{. . n} h . . n$.

(2) Вычислить $(\beta)_{2 n}^{*}=(h . . n)_{2 n}^{*}\left(r_{. . n}\right)_{2 n}^{*}$.

(3) Вычислить $\beta$. Заметим, что $\beta . . n=s . . n$.

Пусть $s_{. . n} f_{. .2 n}=h_{. . n}+x^{n} \gamma$. 
(4) Вычислить $\left(s_{. . n}\right)_{2 n}^{*}$.

(5) Вычислить $\left(s_{. . n} f_{. .2 n}\right)_{2 n}^{*}=\left(s_{. . n}\right)_{2 n}^{*}\left(f_{. .2 n}\right)_{2 n}^{*}$.

(6) Вычислить $s_{. . n} f_{.2 n} \bmod \left(x^{2 n}-1\right)$ и, в частности, $\gamma_{. . n}$.

Пусть $h=h_{. . n}+x^{n} h^{\prime}$ и $\delta=r_{. . n}\left(h_{. . n}^{\prime}-\gamma_{. . n}\right)$.

(7) Вычислить $\left(h_{. . n}^{\prime}-\gamma_{. . n}\right)_{2 n}^{*}$.

(8) Вычислить $(\delta)_{2 n}^{*}=\left(r_{. . n}\right)_{2 n}^{*}\left(h_{. . n}^{\prime}-\gamma_{. . n}\right)_{2 n}^{*}$.

(9) Вычислить $\delta$. Окончательно получаем, что

$$
s_{. .2 n}=s_{. . n}+x^{n} \delta_{. . n} .
$$

Всего на шагах 1-9 выполняется восемь ДПФ порядка $2 n$, остальные действия имеют сложность $O(n)$. Получаем соотношение для сложности деления

$$
D(2 n) \leqslant I(n)+8 F^{\circ}(2 n)+O(n) .
$$

Если инвертирование выполняется методом п. 5.2, то из приведенного соотношения следует, что

$$
D(n) \leqslant 11,75 F(n)+O(n \log \log n) .
$$

Если для инвертирования использовать метод Шёнхаге, то получаем оценку из [12]:

$$
D(n) \leqslant 12,5 F(n)+O(n) .
$$

В последнем случае оценка сложности может быть уменьшена, если воспользоваться идеей из [24]. Заметим, что при использовании $(2 n, n)$-варианта алгоритма Шёнхаге после этапа инвертирования известен вектор $\left(r_{. . n / 2}\right)_{n}^{*}$. Тогда для вычисления произведения $r g \bmod x^{n}$, где $g \in \mathbf{C}[[x]]$, достаточно пяти ДПФ порядка $n$ (против трех ДПФ порядка $2 n)$. Действительно,

$$
\begin{gathered}
r_{. . n}=r_{. . n / 2}+r^{\prime} x^{n / 2}, \\
g_{. . n}=g_{. . n / 2}+g^{\prime} x^{n / 2} .
\end{gathered}
$$

Тогда

$$
(r g)_{. . n}=r_{. . n / 2} g_{. . n / 2}+x^{n / 2}\left(r_{. . n / 2} g^{\prime}+g_{. . n / 2} r^{\prime}\right)_{. . n / 2} \text {. }
$$

Для вычисления по этой формуле достаточно применить ДПФ к многочленам $r^{\prime}, g_{. . n / 2}$, $g^{\prime}$ и восстановить многочлены $r_{. . n / 2} g . . n / 2$ и $r_{. . n / 2} g^{\prime}+g_{. . n / 2} r^{\prime}$.

В алгоритме Карпа-Маркштейна вычисляется два подобных произведения ( $\beta$ и $\delta)$. Указанный прием позволяет заменить пять ДПФ порядка $2 n$, используемых для их вычисления, девятью ДПФ порядка $n$. Соотношение на сложность тогда перепишется в виде

$$
D(2 n) \leqslant I(n)+3 F^{\circ}(2 n)+9 F^{\circ}(n)+O(n),
$$

откуда вытекает оценка

$$
D(n) \leqslant 12 F(n)+O(n) .
$$




\section{2. Алгоритм ван дер Хувена}

Методом $5-2 n$ доказывается вторая оценка теоремы 3. Введем обозначение

$$
h=h_{. . m}+x^{m} h^{\prime} .
$$

Перепишем (5) в виде

$$
s_{. . m+n}=s_{. . m}+x^{m} r_{. . n}\left(h_{. . n}^{\prime}-\left\lfloor s_{. . m} f_{. . m+n} / x^{m}\right\rfloor\right) \bmod x^{m+n} .
$$

Представим $f$ и $s$ в виде

$$
\begin{aligned}
& f=a_{0}+a_{1} x^{n}+a_{2} x^{2 n}+\ldots, \\
& s=d_{0}+d_{1} x^{n}+d_{2} x^{2 n}+\ldots,
\end{aligned}
$$

где $\operatorname{deg} a_{i}, d_{i}<n$. Также введем обозначение

$$
\xi_{i}=a_{i-1}+a_{i} x^{n}
$$

полагая $a_{-1}=0$.

Выберем $k \in \mathbf{N}$ и рассмотрим следующий алгоритм [10]:

(0) Пусть найден многочлен $r_{. . n}$.

$(0.1)$ Вычисляются $\left(r_{. . n}\right)_{2 n}^{*}$ и $(h . . n)_{2 n}^{*}$.

$(0.2)$ Вычисляется $\left(r_{. . n} h . . n\right)_{2 n}^{*}=\left(r_{. . n}\right)_{2 n}^{*}(h . . n)_{2 n}^{*}$.

(0.3) Вычисляется $r_{. . n} h_{. . n}$ и находится $s_{. . n}=d_{0}=\left(r_{. . n} h_{. . n}\right)_{. . n}$.

Далее на каждом $i$-м шаге, $i=1, \ldots, k-1$, находится $s_{. .(i+1) n}$.

(i.1) Вычисляются $\left(\xi_{i}\right)_{2 n}^{*}$ и $\left(d_{i-1}\right)_{2 n}^{*}$.

Введем обозначение

$$
\alpha=\xi_{i} d_{0}+\xi_{i-1} d_{1}+\ldots+\xi_{1} d_{i-1} .
$$

(i.2) Вычисляется $(\alpha)_{2 n}^{*}$, исходя из (22).

(i.3) Вычисляется $\alpha \bmod \left(x^{2 n}-1\right)$.

Введем обозначение

$$
\beta=\left\lfloor h / x^{i n}\right\rfloor-\left\lfloor\alpha / x^{n}\right\rfloor .
$$

(i.4) Вычисляется $\beta . . n$.

(i.5) Вычисляется $(\beta . . n)_{2 n}^{*}$.

(i.6) Вычисляется $\left(r_{. . n} \beta_{. . n}\right)_{2 n}^{*}=\left(r_{. . n}\right)_{2 n}^{*}\left(\beta_{. . n}\right)_{2 n}^{*}$.

(i.7) Вычисляется $r_{. . n} \beta . . n$.

Окончательно получаем, что

$$
d_{i}=\left(r_{. . n} \beta_{. . n}\right)_{. . n} .
$$


На $i$-м шаге, $i>0$, выполняется пять ДПФ порядка $2 n$, дополнительная сложность составляет $O(i n)$ и определяется п. $(i .2)$. Поэтому для сложности алгоритма в целом справедливо соотношение

$$
D(k n) \leqslant(5 k-2) F^{\circ}(2 n)+I(n)+O\left(k^{2} n\right) .
$$

Выбирая

$$
k=\sqrt{\log n}+O(1)
$$

получаем оценку

$$
D(n) \leqslant 10 F(n)+O(n \sqrt{\log n}) .
$$

\section{3. Деление с остатком}

Задачу деления, то есть вычисления частного $s_{. . n}=(h / f)$..n дополним нахождением остатка

$$
t=\left(h . .2 n-s_{. . n} f . . n+1\right) / x^{n} .
$$

Такое определение остатка может показаться несколько странным, однако оно соответствует традиционной постановке задачи деления с остатком многочлена степени $2 n-1$ на многочлен степени $n$.

Пусть $a(x), b(x) \in \mathbf{C}[x], \operatorname{deg} a \leqslant 2 n-1$ и $\operatorname{deg} b=n$. Пусть $q(x)$ и $r(x)-$ соответственно частное и остаток от деления $a(x)$ на $b(x)$ и $\operatorname{deg} q, r<n$. Тогда

$$
a(x)=q(x) b(x)+r(x) .
$$

Через $\tilde{a}(x), \tilde{q}(x), \tilde{b}(x)$ и $\tilde{r}(x)$ обозначим соответственно многочлены $x^{2 n-1} a(1 / x)$, $x^{n-1} q(1 / x), x^{n} b(1 / x)$ и $x^{n-1} r(1 / x)$. Перепишем (23) в виде

$$
\tilde{a}(x)=\tilde{q}(x) \tilde{b}(x)+x^{n} \tilde{r}(x) .
$$

Тогда

$$
\begin{aligned}
& \tilde{q}=(\tilde{a} / \tilde{b})_{. . n}=\left(\tilde{a}_{. . n} / \tilde{b}_{. . n}\right)_{. . n} ; \\
& \tilde{r}=(\tilde{a}-\tilde{q} \tilde{b}) / x^{n}=\left(\tilde{a}_{. .2 n}-\tilde{q}_{. . n} \tilde{b}_{. . n+1}\right) / x^{n},
\end{aligned}
$$

откуда видно, что $\tilde{q}$ и $\tilde{r}$ вычисляются в точности так же, как $s_{. . n}$ и $t$ в формулировке для степенных рядов. Идея сведения (23) к (24) принадлежит Штрассену [25].

Вернемся к задаче деления с остатком степенных рядов. Обозначим сложность нахождения частного и остатка по модулю $x^{n}$ через $D_{1}(n)$.

Теорема 4. Справедливы соотношения

(a) $D_{1}(n) \leqslant 14 F(n)+O(n)$,

(б) $D_{1}(n) \leqslant 12 F(n)+O(n \sqrt{\log n})$. 
Заметим сначала, что алгоритм п. 6.1 остается корректным, если в вычислениях используется не $f . .2 n$, а $f_{.2 n+1}$. Дело в том, что ДПФ порядка $2 n$ можно использовать для умножения многочлена степени $n-1$ на многочлен степени $n$.

Итак, пусть алгоритм п. 6.1 вычисляет $s_{.2 n}$, а также $\left(f_{. .2 n+1}\right)_{2 n}^{*}$ на промежуточном шаге. Дополним его следующим образом.

Положим

$$
t=\left(h_{. .4 n}-s_{. .2 n} f_{. .2 n+1}\right) / x^{2 n}, \quad s_{. .2 n} f_{. .2 n+1}=h_{. .2 n}+x^{2 n} \varepsilon .
$$

(10) Вычислить $\left(s_{. .2 n}\right)_{2 n}^{*}$.

(11) Вычислить $\left(s_{. .2 n} f_{.2 n+1}\right)_{2 n}^{*}=\left(s_{. .2 n}\right)_{2 n}^{*}\left(f_{. .2 n+1}\right)_{2 n}^{*}$.

(12) Вычислить $s_{.2 n} f_{.2 n+1} \bmod \left(x^{2 n}-1\right)=h_{.2 n}+\varepsilon$.

(13) Вычислить $t=\left\lfloor h_{. .4 n} / x^{2 n}\right\rfloor-\varepsilon$.

Для сложности алгоритма деления с остатком справедлива оценка

$$
D_{1}(2 n) \leqslant I(n)+10 F^{\circ}(2 n)+O(n) .
$$

В итоге получаем, что

$$
D_{1}(n) \leqslant 14 F(n)+O(n) .
$$

Лучшую оценку сложности позволяет получить метод, основанный на алгоритме деления из п. 6.2. Дополним этот алгоритм (с поправкой $\operatorname{deg} a_{k-1} \leqslant n$ и соответственно $\left.\operatorname{deg} \xi_{k-1} \leqslant 2 n\right)$ следующим образом.

(k.0) После шага $k-1$ известен многочлен $s_{. . k n}$, а также $\left(d_{i}\right)_{2 n}^{*}$ для всех $i=0,1, \ldots, k-2$ и $\left(\xi_{i}\right)_{2 n}^{*}$ для всех $i=1, \ldots, k-1$. Положим $\xi_{k}=a_{k}$.

$(k .1)$ Вычисляются $\left(d_{k-1}\right)_{2 n}^{*}$ и $\left(\xi_{k}\right)_{2 n}^{*}$. Представим $s_{. . k n} f_{. . k n+1}$ в виде

$$
s_{. . k n} f_{. . k n+1}=h_{. . k n}+x^{k n}\left(c_{0}+c_{1} x^{n}+\ldots+c_{k-1} x^{(k-1) n}\right),
$$

где $\operatorname{deg} c_{i}<n$. Тогда

$$
c_{i}=\left\lfloor\left(\xi_{k} b_{i}+\xi_{k-1} b_{i+1}+\ldots+\xi_{i+1} b_{k-1}\right) / x^{n}\right\rfloor \bmod x^{n} .
$$

Положим

$$
\alpha_{i}=\xi_{k} b_{i}+\xi_{k-1} b_{i+1}+\ldots+\xi_{i+1} b_{k-1} .
$$

(k.2) Вычисляются $\left(\alpha_{i}\right)_{2 n}^{*}, i=0,1, \ldots, k-1$, исходя из (25).

$(k .3)$ Вычисляются все $\alpha_{i}$ и находятся $c_{i}=\left\lfloor\alpha_{i} / x^{n}\right\rfloor . . n$, где $i=0,1, \ldots, k-1$.

Таким образом, используя $k+2$ ДПФ порядка $2 n$ на $k$-м шаге, выводим соотношение

$$
D_{1}(k n) \leqslant 6 k F^{\circ}(2 n)+I(n)+O\left(k^{2} n\right) .
$$

Если в методе Штрассена сложность деления с остатком приближенно равна сложности одного деления и одного умножения, то в модели, основанной на ДПФ, сложность этой операции сопоставима со сложностью двух умножений: справедлива оценка

$$
D_{1}(n) \leqslant 12 F(n)+O(n \sqrt{\log n}) .
$$




\section{7. Квадратный корень}

Рассмотрим задачу вычисления квадратного корня $f=\sqrt{h}, h . .1=1$. Сохраняем обозначение $r=1 / f$. Сложность извлечения квадратного корня по модулю $x^{n}$ обозначим через $Q(n)$.

Алгоритм, основанный на итерации второго порядка, рассматривался Брентом [2], а быстрый способ вычислений был предложен Бернштейном [9], который показал, что извлечение квадратного корня требует не более $11 F(n)+O(n)$ операций. С применением двойного ДПФ оценка сложности стандартного метода может быть понижена до $10 F(n)$. Блочные методы позволяют вычислять квадратный корень асимптотически еще быстрее; в частности, метод 5 - $3 n$ имеет в этом случае сложность $7,5 F(n)$.

Теорема 5. Справедливы соотношения

(a) $Q(n) \leqslant 10 F(n)+O(n)$;

(б) $Q(n) \leqslant 7.5 F(n)+O(n \sqrt{\log n})$.

\section{1. Модификация стандартного метода}

Из (6) при $m=n$ получаем формулу

$$
f_{. .2 n}=f_{. . n}+\frac{\left(h . .2 n-f_{. . n}^{2}\right) r_{. . n}}{2} \bmod x^{2 n}
$$

Рассмотрим следующий алгоритм.

(0) Пусть найдены многочлены $f_{\text {..n }}$ и $r_{. . n / 2}$, и также известен вектор $\left(r_{. . n / 2}\right)_{n}^{*}$.

(1) Вычислить $\left(f_{. . n}\right)_{n, n / 2}^{*}$ и $\left(r_{. . n / 2}\right)_{n, n / 2}^{*}$.

(2) Вычислить $r_{. . n}$ методом п. 5.1, используя двойные ДПФ порядка $(n, n / 2)$.

Последующие шаги реализуют вычисления по формуле (26). Введем обозначение

$$
f_{. . n}^{2}=h_{. . n}+x^{n} \alpha .
$$

(3) Вычислить $\left(f_{. . n}^{2}\right)_{n}^{*}=\left(f_{. . n}\right)_{n}^{*}\left(f_{. . n}\right)_{n}^{*}$.

(4) Вычислить $f_{. . n}^{2} \bmod x^{n}-1=h_{. . n}+\alpha$ и, следовательно, найти $\alpha$.

Пусть $h=h_{. . n}+x^{n} h^{\prime}$. Введем обозначение

$$
\beta=\left(h_{. . n}^{\prime}-\alpha\right) r_{. . n}
$$

(5) Вычислить $\left(r_{. . n}\right)_{2 n}^{*}$ и $\left(h_{. . n}^{\prime}-\alpha\right)_{2 n}^{*}$.

(6) Вычислить $(\beta)_{2 n}^{*}=\left(h_{. . n}^{\prime}-\alpha\right)_{2 n}^{*}\left(r_{. . n}\right)_{2 n}^{*}$.

(7) Вычислить $\beta$. Окончательно получаем, что

$$
f_{. .2 n}=f_{. . n}+x^{n} \beta_{. . n} / 2 \text {. }
$$


В общей сложности, на шагах 1-7 выполняется два двойных ДПФ порядка $(n, n / 2)$, кроме того, по одному ДПФ порядка $n / 2$ и порядка $n$, и три ДПФ порядка $2 n$. Остальные действия имеют линейную сложность. Для сложности этого алгоритма получаем рекуррентное соотношение

$$
Q(2 n) \leqslant Q(n)+3 F^{\circ}(n / 2)+3 F^{\circ}(n)+3 F^{\circ}(2 n)+O(n),
$$

откуда следует, что

$$
Q(n) \leqslant 10,5 F(n)+O(n) .
$$

Понижение сложности по сравнению с алгоритмом из [9] достигается за счет того, что применение двойного ДПФ позволяет выполнять подзадачу инвертирования более быстрым, чем использовался в [9], алгоритмом Шёнхаге.

Далее, на последнем шаге при вычислении $\beta$ можно обойтись пятью ДПФ порядка $n$ вместо трех ДПФ порядка $2 n$ (см. п. 6.1). Это приводит к оценке сложности

$$
Q(n) \leqslant 10 F(n)+O(n) .
$$

\section{2. Асимптотически эффективный метод}

Второе соотношение теоремы 5 доказывается методом $5-3 n$.

Представим $f$ и $h$ в виде

$$
\begin{aligned}
& f=a_{0}+a_{1} x^{n}+a_{2} x^{2 n}+\ldots, \\
& h=d_{0}+d_{1} x^{2 n}+d_{2} x^{4 n}+\ldots,
\end{aligned}
$$

где $\operatorname{deg} a_{i}<n$ и $\operatorname{deg} d_{i}<2 n$. (Разбиение ряда $h$ на блоки длины $2 n$ позволяет сэкономить часть операций.) Введем обозначения

$$
b=r_{. . n}, \quad \chi_{j}=x^{(2-j) n} b, \quad j=0,1,2 .
$$

Выберем $k \in \mathbf{N}$. Пусть $k$ четно. На каждой итерации следующего алгоритма вычисляются очередные $n$ коэффициентов ряда $f$ согласно (6).

(0) Пусть многочлены $f_{. . n}=a_{0}$ и $r_{. . n}=b$ известны.

$(0.1)$ Вычисляются $b_{3 n}^{*}$ (а также $\left(\chi_{j}\right)_{3 n}^{*}$ с дополнительной сложностью $\left.O(n)\right)$ и $\left(d_{j}\right)_{3 n}^{*}$ для всех $j=0,1, \ldots, k / 2-1$.

Далее на каждом $i$-м шаге, $i=1, \ldots, k-1$, находится $f_{. .(i+1) n}$.

(i.1) Вычисляется $\left(a_{i-1}\right)_{3 n}^{*}$.

Положим

$$
\begin{aligned}
& \beta=\left(h_{. .(i+1) n}-f_{. . i n}^{2}\right) r_{. . n}=x^{i n} \alpha, \\
& \gamma=\sum_{2 \lambda+\mu=i} d_{\lambda} \chi_{\mu}-\sum_{\substack{\lambda+\mu+\nu=i \\
\lambda, \mu<i}} a_{\lambda} a_{\mu} \chi_{\nu} .
\end{aligned}
$$

Очевидно, $\operatorname{deg} \gamma<5 n$.

(i.2) Вычисляется $(\gamma)_{3 n}^{*}$, исходя из (27). 
(i.3) Вычисляется $\gamma \bmod \left(x^{3 n}-1\right)$. Многочлен, образованный средними $n$ коэффициентами $\gamma$, совпадает с $2 a_{i}$, то есть

$$
2 a_{i}=\left\lfloor\gamma / x^{2 n}\right\rfloor . . n
$$

Окончательно получаем, что

$$
f_{. .(i+1) n}=f_{. i n}+a_{i} x^{i n} .
$$

На каждом $i$-м шаге, $i>0$, выполняется по два ДПФ порядка $3 n$. Дополнительная сложность определяется п. (i.2). Для алгоритма в целом получаем оценку

$$
Q(k n) \leqslant(2,5 k-1) F^{\circ}(3 n)+Q(n)+O\left(k^{2} n\right) .
$$

Выбирая

$$
k=\sqrt{\log n}+O(1),
$$

получаем оценку

$$
Q(n) \leqslant 7,5 F(n)+O(n \sqrt{\log n}) .
$$

\section{8. Экспонента}

Рассмотрим задачу вычисления экспоненты $f=e^{h}$, где $h_{. .1}=0$. Пусть по-прежнему $r=1 / f$. Сложность выполнения этой операции по модулю $x^{n}$ обозначим через $E(n)$.

В работе [9] Бернштейн получил асимптотическую оценку сложности вычисления экспоненты $17 F(n)$ методом $A-2 n$, а также оценку $19,5 F(n)$, используя модификацию стандартного метода.Заметим, что в [9] для последнего алгоритма из-за неточности указана завышенная оценка $20 F(n)$. Совсем недавно Бостан и Шост [24] улучшили оценку сложности стандартного метода до $16,5 F(n)$. Используя метод 5 - $2 n$ и более общую, чем (8), итерационную формулу, ван дер Хувен [10] получил оценку $14 F(n)$. На самом деле, можно получить лучшие оценки.

Теорема 6. Справедливы соотношения

(a) $E(n) \leqslant 15,5 F(n)+O(n)$,

(б) $E(n) \leqslant 13 F(n)+O(n \log \log n)$.

Для доказательства первого соотношения будет предъявлен модифицированный стандартный алгоритм, в котором используются двойные и (формально) тройные ДПФ. Для доказательства второй оценки используется метод $A-(2 n, n)$.

\section{1. Модификация стандартного метода}

Из формулы (8) в случае $m=n$ следует, что

$$
f_{. .2 n}=f_{. . n}+\left(h . .2 n-\left(\ln f_{. . n}\right) . .2 n\right) f_{. . n} \bmod x^{2 n} .
$$

Вычисление $\left(\ln f_{. . n}\right) . .2 n$ удобно выполнять при помощи формулы (7), подынтегральное выражение в которой согласно (21) (где $m=n-1$ ) может быть вычислено по формуле

$$
\left((\Delta f) . . n-1 / f_{. . n}\right)_{. .2 n-1}=(\Delta h)_{. . n-1}-x^{n-1} r_{. . n}\left\lfloor(\Delta h)_{. . n-1} f_{. . n} / x^{n-1}\right\rfloor \bmod x^{2 n-1}
$$


в силу того, что

$$
\left((\Delta f)_{. . n-1} / f_{. . n}\right)_{. . n-1}=(\Delta h)_{. . n-1} .
$$

Рассмотрим следующий алгоритм.

(0) Пусть найдены многочлены $f_{. . n}$ и $r_{. . n / 2}$, и также известен вектор $\left(r_{. n / 2}\right)_{n}^{*}$.

(1) Вычислить $\left(f_{. . n}\right)_{n, n / 2, n / 2}^{*}$ и $(r . . n / 2)_{n, n / 2}^{*}$.

(2) Вычислить $r_{. . n}$ методом п. 5.1 с использованием двойных ДПФ.

Шаги 3-9 реализуют вычисления по формуле (29). Заметим, что

$$
\left((\Delta h)_{. . n-1} f_{. . n}\right)_{. . n-1}=(\Delta f)_{. . n-1} \text {. }
$$

Пусть

$$
(\Delta h)_{. . n-1} f_{. . n}=(\Delta f)_{. . n-1}+x^{n-1} \gamma .
$$

(3) Вычислить $(\Delta h)_{. . n-1},(\Delta f)_{. . n-1}$.

(4) Вычислить $\left((\Delta h)_{. . n-1}\right)_{n}^{*}$.

(5) Вычислить $\left((\Delta h)_{. . n-1} f_{. . n}\right)_{n}^{*}=\left((\Delta h)_{. . n-1}\right)_{n}^{*}\left(f_{. . n}\right)_{n}^{*}$.

(6) Вычислить $(\Delta h)_{. . n-1} f_{. . n} \bmod \left(x^{n}-1\right)=(\Delta f)_{. . n-1}+\lfloor\gamma / x\rfloor+x^{n-1} \gamma_{. .1}$, откуда находится $\gamma$.

Введем обозначение $\delta=r_{. . n} \gamma$.

(7) Вычислить $(r . . n)_{2 n}^{*},(\gamma)_{2 n}^{*}$.

(8) Вычислить $(\delta)_{2 n}^{*}=\left(r_{. . n}\right)_{2 n}^{*}(\gamma)_{2 n}^{*}$.

(9) Вычислить $\delta$.

Введем обозначение $h=h_{. . n}+x^{n} h^{\prime}$. Тогда

$$
h_{. .2 n}-\left(\ln f_{. . n}\right)_{. .2 n}=x^{n} h_{. . n}^{\prime}+\mathbf{J}\left(x^{n-1} \delta . . n\right)=x^{n} \alpha .
$$

(10) Вычислить $\alpha$.

Введем обозначение $\beta=\alpha f . . n$.

(11) Вычислить $(\alpha)_{n, n / 2, n / 2}^{*}$.

(12) Вычислить $(\beta)_{n, n / 2, n / 2}^{*}=(\alpha)_{n, n / 2, n / 2}^{*}\left(f_{. . n}\right)_{n, n / 2, n / 2}^{*}$.

(13) Вычислить $\beta$. Окончательно получаем, что

$$
f_{.2 n}=f_{. . n}+x^{n} \beta_{. . n} .
$$


Суммарно на шагах 1-13 выполняется три тройных ДПФ порядка $(n, n / 2, n / 2)$, одно двойное ДПФ порядка $(n, n / 2)$, одно ДПФ порядка $n / 2$, два ДПФ порядка $n$ и три ДПФ порядка $2 n$, остальные действия выполняются с линейной сложностью. Для сложности алгоритма получаем рекуррентное соотношение

$$
E(2 n) \leqslant E(n)+8 F^{\circ}(n / 2)+6 F^{\circ}(n)+3 F^{\circ}(2 n)+O(n),
$$

откуда следует, что

$$
E(n) \leqslant 16 F(n)+O(n) .
$$

Используя на последней итерации алгоритма при вычислении $\delta$ прием из [24], как в п. 6.1 и 7.1, получаем оценку

$$
E(n) \leqslant 15,5 F(n)+O(n) .
$$

Согласно замечанию из п. 4.2 тройные ДПФ порядка $(n, n / 2, n / 2)$ в описанном алгоритме можно реализовать при помощи ДПФ порядка $2 n$.

\section{2. Асимптотически эффективный метод}

Выберем $k \in \mathbf{N}$ и рассмотрим следующий алгоритм. Представим $f, r$ и $\Delta h$ в виде

$$
\begin{aligned}
f & =a_{0}+a_{1} x^{n}+a_{2} x^{2 n}+\ldots, \\
r & =b_{0}+b_{1} x^{n}+b_{2} x^{2 n}+\ldots, \\
\Delta h & =d_{0}+d_{1} x^{n-1}+d_{2} x^{2 n-1}+d_{3} x^{3 n-1}+\ldots,
\end{aligned}
$$

где $\operatorname{deg} a_{i}, b_{i}, d_{i}<n$ и $\operatorname{deg} d_{0}<n-1$.

(0) Пусть многочлены $f_{. .2 n}$ и $r_{. . n}=b_{0}$ известны.

(1) Вычисляются $\left(d_{j}\right)_{2 n, n}^{*}$ для всех $j=0,1, \ldots, 2^{k-1}-1$, а также $\left(a_{0}\right)_{2 n, n}^{*},\left(a_{1}\right)_{2 n, n}^{*}$, $\left(b_{0}\right)_{2 n, n}^{*}$.

Далее на каждом $i$-м шаге, $i=2, \ldots, k$, вычисляются $f_{. .2^{i} n}$ и $r_{. .2^{i-1} n}$.

(i.1) Вычисляется $r_{. .2^{i-1} n}$ и $\left(a_{j}\right)_{2 n, n}^{*}$ для всех $j=2^{i-2}, \ldots, 2^{i-1}-1$, методом из п. 5.2 (но при помощи двойных ДПФ).

(i.2) Вычисляются $\left(b_{j}\right)_{2 n, n}^{*}$ для всех $j=2^{i-2}, \ldots, 2^{i-1}-1$.

Введем обозначение

$$
\delta=r_{. .2^{i-1} n}\left\lfloor(\Delta h)_{. .2^{i-1} n-1} f_{. .2^{i-1} n} / x^{2^{i-1} n-1}\right\rfloor .
$$

При выполнении умножения следует учесть граничный эффект (см. п. 4.3.3).

Положим

$$
\tau=\sum_{\lambda+\mu=2^{i-1}-1} d_{\lambda} a_{\mu} .
$$

(i.3) Вычисляется $(\tau)_{2 n}^{*}$, исходя из (30).

(i.4) Вычисляется $\tau$. Положим $\theta=\left\lfloor\tau / x^{n}\right\rfloor$. 
(i.5) Вычисляется $(\theta)_{2 n, n}^{*}$. Тогда

$$
\delta=y_{0}+y_{1} x^{n}+y_{2} x^{2 n}+\ldots,
$$

где

$$
y_{j}=\sum_{\substack{\lambda+\mu+\nu=j+2^{i-1} \\ \lambda, \mu, \nu<2^{i-1} \\ \mu+\nu \geqslant 2^{i-1}}} b_{\lambda} d_{\mu} a_{\nu}+b_{j} \theta .
$$

(i.6) Вычисляются $\left(y_{j}\right)_{2 n, n}^{*}, j=0,1, \ldots, 2^{i-1}-1$, исходя из (31).

(i.7) Вычисляются $y_{j}, j=0,1, \ldots, 2^{i-1}-1$.

(i.8) Вычисляется $\delta_{. .2^{i-1} n}$.

Положим $h=h_{. .2^{i-1} n}+x^{2^{i-1} n} h^{\prime}$ и

$$
x^{2^{i-1} n} h_{. .2^{i-1} n}^{\prime}+\mathbf{J}\left(x^{2^{i-1} n-1} \delta_{. .2^{i-1} n}\right)=x^{2^{i-1} n} \alpha .
$$

Запишем $\alpha$ в виде

$$
\alpha=c_{0}+c_{1} x^{n}+c_{2} x^{2 n}+\ldots,
$$

где $\operatorname{deg} c_{j}<n$.

(i.9) Вычисляются $c_{j}, j=0,1, \ldots, 2^{i-1}-1$.

$(i .10)$ Вычисляются $\left(c_{j}\right)_{2 n}^{*}, j=0,1, \ldots, 2^{i-1}-1$.

Положим $\beta=\alpha f_{. .2}{ }^{i-1} n$, тогда

$$
\beta=z_{0}+z_{1} x^{n}+z_{2} x^{2 n}+\ldots,
$$

где

$$
z_{j}=\sum_{\substack{\lambda+\mu=j \\ \lambda, \mu<2^{i-1}}} c_{\lambda} a_{\mu} .
$$

(i.11) Вычисляются $\left(z_{j}\right)_{2 n}^{*}, j=0,1, \ldots, 2^{i-1}-1$, исходя из (32).

(i.12) Вычисляются $z_{j}, j=0,1, \ldots, 2^{i-1}-1$.

(i.13) Вычисляется $\beta_{. .2^{i-1} n}$. Окончательно получаем, что

$$
f_{. .2^{i} n}=f_{. .2^{i-1} n}+x^{2^{i-1} n} \beta_{. .2^{i-1} n} .
$$

Оценим сложность вычислений на $i$-м шаге. В п. $(i .1),(i .2),(i .5)$ и $(i .7)$ выполняется соответственно $2^{i-1}+2,2^{i-2}, 1$, и $2^{i-1}$, то есть всего $1,25 \cdot 2^{i}+3$, двойных ДПФ порядка $(2 n, n)$. В п. $(i .3),(i .10),(i .12)$ вычисляется $2^{i}+1$ ДПФ порядка $2 n$. П. $(i .6)$ и $(i .11)$ выполняются со сложностью $O\left(n M\left(2^{i}\right)\right)$, а остальные действия - со сложностью $O\left(2^{i} n\right)$. Для алгоритма в целом получаем оценку

$$
E\left(2^{k} n\right) \leqslant\left(5 \cdot 2^{k}+4 k-10\right) F^{\circ}(2 n)+\left(3 \cdot 2^{k}+3 k-5\right) F^{\circ}(n)+E(2 n)+O\left(2^{k} k n\right) .
$$


При выборе

$$
k=\log \log n+O(1)
$$

получаем, что

$$
E(n) \leqslant 13 F(n)+O(n \log \log n) .
$$

Асимптотически такую же оценку сложности (но с худшим остаточным членом) можно получить и методом 5 - $(2 n, n)$.

\section{9. Возведение в степень}

Очевидный способ реализации операции возведения ряда $h, h . .1=1$, в произвольную степень $C$ состоит в композиции логарифмирования, умножения на $C$ и вычисления экспоненты:

$$
h^{C}=e^{C \ln h} .
$$

Поэтому сложность $P(n)$ возведения в степень очевидно оценивается так:

$$
P(n) \leqslant D(n)+E(n)+O(n) .
$$

Эта общая оценка может быть уточнена при использовании специальных алгоритмов для логарифма и экспоненты, имеющих общую часть. Например, при возведении в степень с помощью алгоритмов из п. 6.1 и 8.1 можно сэкономить порядка $0,5 F(n)$ операций (для выполнения шага 4 на последней итерации в алгоритме п. 8.1 не нужно ДПФ) общая сложность при этом составит $27 F(n)+O(n)$. Комбинация лучших описанных алгоритмов (п. 6.2, 8.2) приводит к асимптотической оценке $23 F(n)$. Еще лучшую оценку, $20,5 F(n)$, можно получить вариантом метода $5-(2 n, n)$, организуя итерационный процесс непосредственно для операции возведения в степень.

\section{Теорема 7. Справедливы соотношения}

(a) $P(n) \leqslant 27 F(n)+O(n)$,

(б) $P(n) \leqslant 20,5 F(n)+O(n \sqrt{\log n \log \log n})$.

Остается доказать второе соотношение. Введем обозначения

$$
r=1 / f, \quad \rho=1 / h, \quad s=\Delta h / h=(1 / C) \Delta f / f .
$$

Воспользуемся формулой (9). Согласно (7) и (21),

$$
\begin{aligned}
& \left(\Delta\left(C \ln h-\ln f_{. . m}\right)\right)_{. . m+n-1} \\
& =\left(C s-\Delta \ln f_{. . m}\right)_{. . m+n-1} \\
& =C\left(\rho_{. . n}\left(\Delta h-s_{. . m-1} h_{. . m+n}\right)+x^{m-1} r_{. . n}\left\lfloor s_{. . m-1} f_{. . m} / x^{m-1}\right\rfloor\right)_{. . m+n-1} .
\end{aligned}
$$

Представим $f, r, s, \Delta h, h, \rho$ в виде

$$
\begin{aligned}
f & =a_{0}+a_{1} x^{n}+a_{2} x^{2 n}+\ldots, \\
r & =b_{0}+b_{1} x^{n}+b_{2} x^{2 n}+\ldots, \\
s & =c_{0}+c_{1} x^{n-1}+c_{2} x^{2 n-1}+c_{3} x^{3 n-1}+\ldots, \\
\Delta h & =d_{0}+d_{1} x^{2 n-1}+d_{2} x^{4 n-1}+d_{3} x^{6 n-1}+\ldots, \\
h & =u_{0}+u_{1} x^{n}+u_{2} x^{2 n}+\ldots, \\
\rho & =v_{0}+v_{1} x^{n}+v_{2} x^{2 n}+\ldots,
\end{aligned}
$$


где степени $a_{i}, b_{i}, c_{i}, d_{i}, u_{i}, v_{i}$ меньше $n$ и $\operatorname{deg} c_{0}, \operatorname{deg} d_{0}<n-1$. Пусть $k, l \in \mathbf{N}$ и $l$ четно. Для построения алгоритма мы используем несколько усложненную версию метода 5 - $(2 n, n)$, в которой на каждой итерации вычисляются очередные $n l$ коэффициентов искомого ряда $f$ : это связано с необходимостью учета граничного эффекта при вычислении тройного произведения $r_{. . n}\left\lfloor s_{. . m-1} f . . m / x^{m-1}\right\rfloor$ (см. п. 4.3.3).

(0) Пусть $f_{. l n}, r_{. l n}, \rho_{. l n}, s_{. . \ln -1}$ известны.

(1) Вычисляются $\left(d_{j}\right)_{2 n, n}^{*}$ для всех $j=0,1, \ldots, k l / 2-1,\left(u_{j}\right)_{2 n, n}^{*}$ для всех $j=0,1, \ldots, k l-1$, а также $\left(b_{j}\right)_{2 n, n}^{*}$ и $\left(v_{j}\right)_{2 n, n}^{*}$ для всех $j=0,1, \ldots, l-1$.

Далее на каждом $i$-м шаге, $i=2, \ldots, k$, следуя (33), находятся очередные $n l$ коэффициентов рядов $f$ и $s$.

Для того, чтобы вычислить $S_{. . i l n-1}$, выполняются следующие операции:

(i.1) Вычисляются $\left(c_{j}\right)_{2 n, n}^{*}$ для всех $j=(i-2) l, \ldots,(i-1) l-1$.

(i.2) Далее $s_{. . i l n-1}$ восстанавливается из тройного произведения

$$
s_{. i \ln -1}-s_{. .(i-1) \ln -1}=\rho_{. . \ln }\left(\Delta h-s_{. .(i-1) \ln -1} h_{. i \operatorname{lln}}\right) \bmod x^{i \ln -1} .
$$

Сложность пункта определяется $l$ обратными ДПФ порядка $(2 n, n)$.

Для того, чтобы вычислить $\Delta \ln f_{. . i l n}$, выполняются следующие операции:

(i.3) Вычисляются $\left(a_{j}\right)_{2 n, n}^{*}$ для всех $j=(i-2) l, \ldots,(i-1) l-1$.

(i.4) Далее $\Delta \ln f . . i \ln$ восстанавливается из тройного произведения

$$
\begin{aligned}
& \Delta \ln f_{. .(i-1) \ln }-\Delta \ln f_{. . i \ln }
\end{aligned}
$$

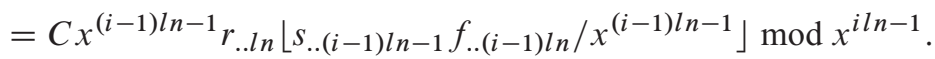

Сложность пункта определяется $l$ обратными ДПФ порядка $(2 n, n)$, а также обратным ДПФ порядка $2 n$ и ДПФ порядка $(2 n, n)$, выполняемыми с целью учета граничного эффекта аналогично шагам (i.3)-(i.5) алгоритма п. 8.2.

(i.5) После вычисления формальным интегрированием $(C \ln h-\ln f . .(i-1) \ln )$..iln остается найти $f_{. . i l n}$ из произведения (9), для чего достаточно выполнить по $l$ прямых и обратных ДПФ порядка $2 n$.

Дополнительную сложность (то есть без учета ДПФ) каждого из пунктов можно оценить как $O(n M(i l))$. Для сложности алгоритма получаем оценку

$$
\begin{aligned}
P(k \ln ) \leqslant(7,5 k l-4 l+2 k-2) F^{\circ}(2 n)+ & (5,5 k l-2 l+k-1) F^{\circ}(n) \\
+ & P(\ln )+2 I(\ln )+D(\ln )+O(k n M(k l)),
\end{aligned}
$$

из которой при выборе

$$
k, l=\sqrt{\log n / \log \log n}+O(1)
$$

получаем оценку

$$
P(n) \leqslant 20,5 F(n)+O(n \sqrt{\log n \log \log n}) .
$$


Описанный алгоритм при натуральных значениях показателя $C$ интересно сравнить со стандартным методом возведения в степень, в котором последовательно при помощи умножений и возведений в квадрат вычисляются все большие и большие степени, пока не будет получена искомая. Легко видеть, что сложность стандартного метода оценивается величиной $2 l(C)(F(2 n)+O(n))$, где $l(C)$ - длина минимальной аддитивной цепочки для $C$. Эта оценка лучше полученной выше, если $l(C) \leqslant 5$, то есть при $C \leqslant 18$, а также при $C=20,24,32$.

\section{0. Замечания}

\section{1. О сложности решения линейных однородных дифференциальных уравнений}

Рассмотрим задачу нахождения приближенного (по модулю $x^{n}$ ) решения линейного однородного дифференциального уравнения (ЛОДУ) порядка $p$ :

$$
\Delta^{p} f=a_{p-1} \Delta^{p-1} f+\ldots+a_{1} \Delta f+a_{0} f
$$

с начальными условиями

$$
\left(\Delta^{i} f\right)_{. .1}=v_{i}, \quad i=0,1, \ldots, p-1,
$$

где $a_{i}, f \in \mathbf{C}[[x]], v_{i} \in \mathbf{C}$. Эта задача может быть переписана в матричной форме:

$$
\begin{aligned}
\Delta F & =\left[\begin{array}{ccccc}
a_{p-1} & a_{p-2} & \ldots & a_{1} & a_{0} \\
1 & 0 & \ldots & 0 & 0 \\
0 & 1 & \ldots & 0 & 0 \\
\ldots \ldots & \ldots & \ldots & \ldots & \ldots \\
0 & 0 & \ldots & 1 & 0
\end{array}\right] F, \\
F_{. .1} & =V
\end{aligned}
$$

где $F \in \mathbf{C}[[x]]^{p}, V=\left(v_{p-1}, \ldots, v_{0}\right)^{T}$. (Обозначение $g_{. . n}=g \bmod x^{n}$ распространяется также на векторы и матрицы степенных рядов.) Последняя задача является частным случаем задачи

$$
\Delta F=A F, \quad F_{. .1}=V
$$

с произвольной матрицей $A$. С задачей (36) тесно связана задача нахождения фундаментальной системы решений, то есть вычисление матрицы $M$ такой, что

$$
\Delta M=A M, \quad M_{. .1}=E,
$$

где $E-$ единичная матрица.

Сложность решения задач (34), (36), (37) методом последовательных приближений оценивалась в работах $[19,10]$. В [19] было показано, что задача (37) решается со сложностью $O\left(F(n) p^{2}\right)$, задача (36) - со сложностью $O\left(F(n) p^{2} \log n\right)$, а исходная задача (34) - со сложностью $O\left(F(n) p^{2}\right)$. Эти оценки были уточнены в [10]. Пусть $q$ обозначает число отличных от констант элементов матрицы $A$. Тогда из метода [10] следуют асимптотические верхние оценки: для задачи $(37)-\left(2 q+12 p^{2}\right) F(n)$, для задачи (36) - 
$(2 q+12 p) F(n)$ (в самой работе [10] указана более слабая оценка $(6 q+8 p) F(n))$. Из последней оценки вытекает (при подстановке $q=p$ ) оценка сложности решения задачи (34) в виде $14 p F(n)$. Везде предполагается, что $p$ достаточно мало по сравнению с $n$.

В работе [10] методом $E-2 n$ реализуются итерации

$$
\begin{aligned}
M_{. . m+n} & =M_{. . m}-M_{. . n} \mathbf{J}\left(M_{. . n}^{-1}\left((\Delta M) . . m-1-A_{. . m+n-1} M_{. . m}\right)\right), \\
F_{. . m+n} & =F_{. . m}-M_{. . n} \mathbf{J}\left(M_{. . n}^{-1}\left((\Delta F)_{. . m-1}-A_{. . m+n-1} F_{. . m}\right)\right)
\end{aligned}
$$

для задач (37) и (36) соответственно. В этом способе решение задачи (37) используется для решения задачи (36).

Для нахождения очередных блоков коэффициентов длины $n$ по формуле (38) методом 5 - $2 n$ используется следующая последовательность вычислений:

(1) Вычисляется необходимый блок произведения $G=A_{. . m+n-1} F_{. . m}$, для чего выполняется $q+2 p$ ДПФ порядка $2 n$.

(2) Вычисляется необходимый блок произведения $H=M_{. . n}^{-1} G$ посредством $2 p$ ДПФ порядка $2 n$.

(3) Окончательно, вычисляется необходимый блок произведения $M_{. . n} \mathbf{J} H$ также с использованием $2 p$ ДПФ порядка $2 n$.

При оценке сложности используется тот факт, что ДПФ для элементов матриц $M$, $M^{-1}$ вычисляются только на первом шаге.

Из (38) видно, что вычисление блоков произведений $G$ и $H$ можно заменить вычислением блока в тройном произведении $M_{. . n}^{-1}\left\lfloor A_{. . m+n-1} F_{. . m} / x^{m-1}\right\rfloor$. Для этой цели можно использовать метод $5-(2 n, n)$ с двойным разбиением рядов, как в алгоритме возведения в степень из раздела 9. Сложность выделения необходимого блока длины $n$ из указанного произведения на шаге итерации соответствует сложности $q+2 p$ двойных ДПФ порядка $(2 n, n)$.

Таким образом, сложность решения задачи (36) методом 5 -( $2 n, n)$ составляет асимптотически $(3 q+10 p) F(n)$. Как следствие, сложность решения ЛОДУ (34) не превосходит асимптотически $13 p F(n)$. Аналогично для сложности задачи (37) можно получить оценку $\left(3 q+10 p^{2}\right) F(n)$.

Оценка $14 p F(n)$ сложности решения ЛОДУ порядка $p$ методом $5-2 n$ является грубой, так как. матрица $A$ в приведенной задаче (35) имеет специальный вид. Вновь рассмотрим итерацию (38), где матрица $A$ задана соотношением (35). Так как только одна компонента произведения $G$ является нетривиальной, для его вычисления достаточно выполнить $2 p+1$ ДПФ порядка $2 n$. Как следствие, $p+1$ ДПФ достаточно для вычисления $H$. Наконец, достаточно найти только одну компоненту вектора $F$, поэтому заключительное умножение также можно выполнить, используя $p+1$ ДПФ. Таким образом, для сложности решения ЛОДУ (34) справедлива асимптотическая оценка $(8 p+6) F(n)$ (она лучше, чем в методе $5-(2 n, n)$, при $p \geqslant 2)$. Аналогичные рассуждения позволяют для сложности нахождения фундаментальной системы решений уравнения (34) указать оценку $\left(6 p^{2}+8 p\right) F(n)$. 


\section{2. О мультипликативной сложности}

В качестве меры сложности операций можно рассматривать мультипликативную сложность, в которой учитываются только нескалярные умножения и деления. Известно (см., например, [17]), что мультипликативная сложность умножения равна

$$
M^{*}(n)=2 n-1 .
$$

Инвертирование по формуле (5) реализуется с мультипликативной сложностью $4 n+O(1)$, а всего в соответствующем методе выполняется асимптотически $10 F(n)$ операций (см. [9]). Если использовать итерацию третьего порядка

$$
r_{. .3 n}=r_{. . n}\left(1+\left(2-r_{. . n} f_{. .3 n}\right)\left(1-r_{. . n} f_{. .3 n}\right)\right),
$$

то можно указать метод с мультипликативной сложностью также $4 n$, но с общей сложностью 9,5F $(n)$. Вариант этого же метода (см. [17]) имеет меньшую мультипликативную сложность $3,75 n$ и общую сложность $9,75 F(n)$. Можно также предъявить метод, основанный на итерации четвертого порядка, с мультипликативной сложностью $4 n$ и общей сложностью $(28 / 3) F(n)$. (Формулы для итераций высших порядков см., например, в упражнении 9.11 в [3].)

Мультипликативная сложность деления обычно оценивается, исходя из соотношения

$$
D^{*}(n) \leqslant I^{*}(n)+M^{*}(n),
$$

откуда следует, что

$$
D^{*}(n) \leqslant 5,75 n
$$

(общая сложность соответствующего алгоритма 15,75F(n)). Из метода п. 6.1 следует, что

$$
D^{*}(n) \leqslant I^{*}(n / 2)+3 n \leqslant 4,875 n,
$$

при этом общая сложность алгоритма оценивается величиной $12,875 F(n)$. Соответственно для деления с остатком (см. п. 6.3, первый метод)

$$
D_{1}^{*}(n) \leqslant D^{*}(n)+n \leqslant 5,875 n .
$$

Эта оценка была известна Шёнхаге в 1999 г.

В заключение отметим, что квадратный корень степенного ряда можно вычислить при помощи итерации (26) с мультипликативной сложностью $5 n$ и общей сложностью $11 F(n)$ методом [9], а экспоненту - с мультипликативной сложностью $7 n$ и общей сложностью $17 F(n)$ вариантом метода п. 8.1. Мультипликативную сложность возведения в степень удобно оценивать, исходя из соотношения

$$
P^{*}(n) \leqslant D^{*}(n)+E^{*}(n),
$$

откуда следует, что

$$
P^{*}(n) \leqslant 11,875 n,
$$

а общая сложность соответствующего алгоритма $-29,375 F(n)$.

В табл. 1 сведены результаты о сложности обсуждавшихся операций. Заметим, что аналогичные оценки имеют место для рядов с коэффициентами из произвольного алгебраически замкнутого поля, а также из произвольного кольца (при переопределении $F(n)$ в последнем случае, см. [4]).

Автор благодарен научному руководителю С. Б. Гашкову за внимание к работе и ряд ценных замечаний. 
Таблица 1.

\begin{tabular}{|c|c|c|c|c|c|}
\hline Операция & $F(n):$ & асимпт. & метод & станд. & C \\
\hline ДПФ & & 1 & - & 1 & $1,5 n \log n$ \\
\hline Умножение & & 6 & - & 6 & $9 n \log n$ \\
\hline Инвертирование & & 7,5 & $A-3 n$ & 9 & $11,25 n \log n$ \\
\hline Деление и логарифм & & 10 & $B-2 n[10]$ & 12 & $15 n \log n$ \\
\hline Деление с остатком & & 12 & $B-2 n[10]$ & 14 & $18 n \log n$ \\
\hline Квадратный корень & & 7,5 & $B-3 n$ & 10 & $11,25 n \log n$ \\
\hline Экспонента & & 13 & $A-(2 n, n)$ & 15,5 & $19,5 n \log n$ \\
\hline Возведение в степень & & 20,5 & $B-(2 n, n)$ & 27 & $30,75 n \log n$ \\
\hline ЛОДУ порядка 1 & & 13 & $B-(2 n, n)$ & - & $19,5 n \log n$ \\
\hline ЛОДУ порядка $p$ & & $8 p+6$ & $B-2 n[10]$ & - & $(12 p+9) n \log n$ \\
\hline
\end{tabular}

\section{Список литературы}

1. Cook S., On the minimum computation time of functions, Doctoral Thesis. Harvard Univ., Cambridge, Mass., 1966.

2. Brent R. P., Multiple-precision zero-finding methods and the complexity of elementary function evaluation. In: Analytic Computational Complexity (Traub J. F., ed.). Academic Press, New York, 1975, pp. 151-176.

3. von zur Gathen J., Gerhard J., Modern computer algebra. Cambridge Univ. Press, Cambridge, 1999.

4. Bernstein D. J., Fast multiplication and its applications. In: Algorithmic Number Theory. Lattices, Number Fields, Curves and Cryptography (Mathematical Sciences Research Institute Publications), 44 (Buhler J. P. et al., eds.). Cambridge, 2008, pp. 325-384.

5. Sieveking M., An algorithm for division of power series. Computing (1972) 10, 153-156.

6. Brent R. P., Kung H. T., Fast algorithms for manipulating formal power series. J. Assoc. Comput. Mach. (1978) 25, №4, 581-595.

7. Aho A. V., Hopcroft J. E., Ullman J. D., The design and analysis of computer algorithms. AddisonWesley, Reading, Mass., 1976.

8. Schönhage A., Variations on computing reciprocals of power series. Inf. Process. Lett. (2000) 74, 41-46.

9. Bernstein D. J., Removing redundancy in high-precision newton iteration, http://cr.yp.to/fastnewton.html (не опубликовано).

10. van der Hoeven J., Newton's Method and FFT Trading, Technical Report 2006-17. Univ. Paris-Sud, Orsay, 2006.

11. Карацуба А. А., Офман Ю. П., Умножение многозначных чисел на автоматах. Докл. АН СССР (1962) 145, №2, 293-294.

12. Hanrot G., Quercia M., Zimmermann P., The middle product algorithm. I: Speeding up the division and square root of power series. Appl. Algebra Eng. Commun. Comput. (2004) 14, 415-438.

13. Сергеев И. С., Быстрые алгоритмы для элементарных операций со степенными рядами. В сб.: Материаль IX Международного семинара «Дискретная математика и ее приложения». Издво мех.-мат. ф-та МГУ, Москва, 2007, с. 123-126.

14. Bernstein D. J., The tangent FFT. Lecture Notes Computer Sci. (2007) 4851, 291-300.

15. Schönhage A., Asymptotically fast algorithms for the numerical multiplication and division of polynomials with complex coefficients. Lecture Notes Computer Sci. (1982) 144, 3-15. 
16. van der Hoeven J., Notes on the truncated fourier transform, Technical Report 2005-5. Univ. ParisSud, Orsay, 2005.

17. Bürgisser P., Clausen M., Shokrollahi M. A., Algebraic complexity theory. Springer, Berlin, 1997.

18. Knuth D. E., The art of computer programming: seminumerical algorithms, 2. Addison-Wesley Longman, Boston, 1998.

19. Bostan A., Chyzak F., Ollivier F., Salvy B., Schost É., Sedoglavic A., Fast computation of power series solutions of systems of differential equations. In: Proc. Symposium on Discrete Algorithms. SIAM, Philadelphia, 2007, pp. 1012-1021.

20. Schulz G., Iterative Berechnung der reziproken Matrix. Z. Angewandte Math. Mech. (1933) 13, 57-59.

21. Cooley J. W., Tukey J. W., An algorithm for the machine calculation of complex Fourier series. Math. Comput. (1965) 19, 297-301.

22. van der Hoeven J., New algorithms for relaxed multiplication. J. Symbolic Comput. (2007) 42, 792-802.

23. Karp A. H., Markstein P., High-precision division and square root. ACM Trans. Math. Softw. (1997) 23, 561-589.

24. Bostan A., Schost É., A simple and fast algorithm for computing exponentials of power series. Inf. Process. Lett. (2009) 109, 754-756.

25. Strassen V., Die Berechnungskomplexität von elementarsymmetrischen Funktionen und von Interpolationskoeffizienten. Numer. Math. (1973) 20, 238-251.

Статья поступила 2.08.2008. 